TRANSACTIONS OF THE

AMERICAN MATHEMATICAL SOCIETY

Volume 361, Number 10, October 2009, Pages 5111-5137

S 0002-9947(09)04729-1

Article electronically published on May 5, 2009

\title{
LOCATING THE FIRST NODAL SET IN HIGHER DIMENSIONS
}

\author{
SUNHI CHOI, DAVID JERISON, AND INWON KIM
}

\begin{abstract}
We extend the two-dimensional results of Jerison (2000) on the location of the nodal set of the first Neumann eigenfunction of a convex domain to higher dimensions. If a convex domain $\Omega$ in $\mathbb{R}^{n}$ is contained in a long and thin cylinder $[0, N] \times B_{\epsilon}(0)$ with nonempty intersections with $\left\{x_{1}=0\right\}$ and $\left\{x_{1}=N\right\}$, then the first nonzero eigenvalue is well approximated by the eigenvalue of an ordinary differential equation, by a bound proportional to $\epsilon$, whose coefficients are expressed in terms of the volume of the cross sections of the domain. Also, the first nodal set is located within a distance comparable to $\epsilon$ near the zero of the corresponding ordinary differential equation.
\end{abstract}

\section{INTRODUCTION}

Let $\Omega$ be a bounded convex domain in $\mathbb{R}^{n}$. Let $u$ be an eigenfunction for $\Omega$ associated with the smallest nonzero eigenvalue $\lambda$ of the Neumann problem for $\Omega$, that is,

$$
\Delta u=-\lambda u \text { in } \Omega, u_{\nu}=0 \text { on } \partial \Omega,
$$

where $u_{\nu}=\nu \cdot \nabla u$ and $\nu$ denotes the outer normal unit vector at each point on $\partial \Omega$. The purpose of this paper is to locate the first nodal set $\Lambda=\{u=0\}$ and to estimate the first nonzero eigenvalue $\lambda$. We show that $\Lambda$ is near the unique zero of the first nonconstant eigenfunction of a certain ordinary differential equation, and we estimate the difference between $\lambda$ and the first nonzero eigenvalue of the corresponding ordinary differential equation.

Assume

$$
\Omega \subset\left\{\left(x, y_{1}, \ldots, y_{n-1}\right): x \in[0, N],\left(y_{1}, \ldots, y_{n-1}\right) \in B_{\epsilon}(0)\right\} \subset \mathbb{R}^{n}
$$

and suppose further that

$$
\Omega(s):=\Omega \cap\{x=s\} \text { is nonempty for } 0<s<N .
$$

Let $u$ be the first nonconstant eigenfunction for $\Omega$. Denote by $\phi_{1}$ the first nonconstant eigenfunction with the smallest nonzero eigenvalue $\mu_{1}$ for the Neumann problem on $[0, N]$ given by

$$
-\left(w \phi_{1}^{\prime}\right)^{\prime}=\mu_{1} w \phi_{1} \text { on }(0, N) ; w(s) \phi_{1}^{\prime}(s) \rightarrow 0 \text { as } s \rightarrow 0^{+} \text {or } s \rightarrow N^{-},
$$

Received by the editors March 21, 2007.

2000 Mathematics Subject Classification. Primary 35J25; Secondary 35J05.

Key words and phrases. Convex domains, eigenfunctions.

The first author was partially supported by NSF grant DMS 0713598.

The second author was partially supported by NSF grant DMS 0244991.

The third author was partially supported by NSF grant DMS 0627896. 
where $w(s)$ is the $(n-1)$-dimensional volume of $\Omega(s)$. Let $s_{1} \in(0, N)$ be the unique zero of $\phi_{1}$, i.e., $\phi_{1}\left(s_{1}\right)=0$. The main results of this paper are as follows.

Theorem 1.1. If $u$ is the first nonconstant Neumann eigenfunction of $\Omega$ and $\Omega$ satisfies (1.2) and (1.3), then there is a dimensional constant $C$ such that

(a) $u\left(x, y_{1}, \ldots, y_{n-1}\right)=0$ implies $\left|x-s_{1}\right|<C \epsilon$,

(b) $(1-C \epsilon / N) \mu_{1} \leq \lambda \leq \mu_{1}$,

where $s_{1}$ and $\mu_{1}$ are given in (1.4).

Theorem 1.1 was proven by Jerison ([J1]) for $n=2$. By taking a new coordinate system, he bounds the first eigenvalue $\lambda$ from below by a formula whose coefficients are expressed in terms of the width of the cross section $\Omega(x)=\{y:(x, y) \in$ $\Omega$. (Here $y$-axis is chosen so that the projection of $\Omega$ onto the $y$-axis has the shortest possible length.) Using ODE eigenvalue estimates, he first locates the nodal set, and then, using the location of the first nodal set, he estimates the first eigenvalue. However for $n>2$, a parallel approach leads to a weaker result mainly due to the fact that we do not have a proper coordinate system $\left(s, t_{1}, \ldots, t_{n-1}\right) \rightarrow$ $\left(x, y_{1}, \ldots, y_{n-1}\right)$ satisfying

$$
\int_{0}^{N}\left|\partial_{s} y_{j}\right| d s \leq C \epsilon, \quad j=1, \ldots, n-1 .
$$

(See the Remarks at the end of this section.)

In this paper we extend results in J1 to higher dimensions, with a different approach to the problem. We first estimate the difference between the eigenvalues of the original PDE and the corresponding ODE by taking a one-dimensional test function and using a sharp result of Kröger on the upper bound of a gradient of a Neumann eigenfunction and also by using a new coordinate system which will be constructed in section 4 . (We will only need a pointwise estimate (see (II') in section 4) weaker than (1.5).) Based on these estimates, it turns out that we can find a bound on the width of the first nodal set. Once we prove that the nodal set is thin, i.e., the diameter of the nodal set is comparable to $\epsilon$, then we are able to locate the nodal set near the zero of the eigenfunction of the corresponding ODE.

First, we derive the second inequality of (b). Recall that $u$ minimizes the Dirichlet integral

$$
J(v)=\int_{\Omega}|\nabla v|^{2}
$$

among all functions $v$ on $\Omega$ satisfying

$$
\int_{\Omega} v^{2}=1, \int_{\Omega} v=0
$$

The minimum value of $J$ is the eigenvalue $\lambda$. If we consider functions of $x$ alone, i.e., $v\left(x, y_{1}, \ldots, y_{n-1}\right)=\phi(x)$, then

$$
J(v)=I(\phi):=\int_{0}^{N} \phi^{\prime}(s)^{2} w(s) d s
$$

and the constraints (1.7) become

$$
\int_{0}^{N} \phi(s)^{2} w(s) d s=1, \int_{0}^{N} \phi(s) w(s) d s=0 .
$$


As in [J1] we observe that the minimizer of (1.8) under the constraints (1.9) is the first nonzero eigenfunction $\phi_{1}$ given in (1.4) and $I\left(\phi_{1}\right)=\mu_{1}$. Hence

$$
\lambda \leq \mu_{1} \text {. }
$$

Remark 1. If we normalize $N=1$, then by [L] and [ZY], $C_{1} \leq \lambda$ for some absolute constant $C_{1}>0$, and by plugging in the test function $\phi(x)=\sin \pi x_{1}$, we get $\lambda \leq C_{2}$ for some dimensional constant $C_{2}$.

Remark 2. In the case of the Dirichlet problem on a planar convex domain, Jerison J2 obtained results corresponding to Theorem 1.1. Later Grieser and Jerison (GJ1], GJ2]) showed that the nodal line is in an $x$-interval of much shorter length $C \epsilon / N$ (possibly at distance $C \epsilon$ from $s_{1}$ ). We expect that there is an analogous bound in the Neumann problem.

Remark 3. An analogous approach to the method in [J1 for higher dimensions, by modifying the methods in [J1], JJ2, leads to a result weaker than Theorem 1.1, i.e., with $\epsilon \log (N / \epsilon)$ instead of $\epsilon$.

\section{Preliminary Results and COROllaries}

Throughout the paper we normalize $\Omega$ such that $N=1$. As mentioned in the Introduction, a key ingredient in the proof of Theorem 1.1 is Kröger's comparison theorems on the gradient of eigenfunction $u$, which we state below.

Theorem 2.1 (Kröger, $\mathrm{K} 1], \mathrm{BQ}$ ). Let $\Omega$ be a convex domain in $\mathbb{R}^{n}$ with smooth boundary. Let $u$ be the first eigenfunction for the Laplace operator on $\Omega$ with the associated eigenvalue $\lambda>0$, under Neumann boundary conditions. Let $v$ be a solution on some interval $(a, b)$ of the differential equation

$$
v^{\prime \prime}(x)+\frac{n-1}{x} v^{\prime}(x)=-\lambda v(x) \text { on }(a, b) \quad v^{\prime}(a)=v^{\prime}(b)=0
$$

such that $v^{\prime} \neq 0$ on $(a, b)$ and $[\min u, \max u] \subset[\min v, \max v]$. Then

$$
\left|\nabla\left(v^{-1} \circ u\right)\right| \leq 1 \text {. }
$$

Theorem 2.2 (Kröger, [K2]). Let $\Omega, u$ and $\lambda$ be given as in Theorem 2.1 . Suppose $\max u \geq-\min u$. Let $b$ be a positive number such that $\lambda$ is the first nonzero eigenvalue of

$$
\psi^{\prime \prime}(x)+\frac{n-1}{x} \psi^{\prime}(x)=-\lambda \psi(x) \text { on }[0, b], \quad \psi^{\prime}(0)=\psi^{\prime}(b)=0 .
$$

If $\psi$ is the corresponding eigenfunction with $\psi(0)>0$, then

$$
\frac{\max u}{-\min u} \leq \frac{\max \psi}{-\min \psi}
$$

Corollary 2.3. Let $\Omega$ and $u$ be given as in Theorem 2.1. If $N=1$ and $\max |u|=1$, then $|\nabla u| \leq C$ for some dimensional constant $C$.

Proof. First, we claim that if $0 \leq a<b$, then the solution $v$ to (2.1) satisfies

$$
\max \left|v^{\prime}\right| \leq \sqrt{\lambda}|v(a)|
$$

To see this, multiply $v^{\prime}$ to both sides of (2.1) to obtain

$$
v^{\prime} v^{\prime \prime}+\lambda v^{\prime} v=\frac{1}{2}\left(\left(v^{\prime}\right)^{2}+\lambda v^{2}\right)^{\prime}=-\frac{n-1}{x}\left(v^{\prime}\right)^{2} \leq 0
$$


for $x>0$. Hence,

$$
\left(v^{\prime}\right)^{2}(x)+\lambda v^{2}(x) \leq\left(v^{\prime}\right)^{2}(a)+\lambda v^{2}(a)=\lambda v^{2}(a),
$$

and the claim follows.

Now, for sufficiently large $M>0$, consider an interval $(a, b) \subset(M, \infty)$ such that $\lambda$ is the first nonzero eigenvalue of the Neumann problem (2.1). Let $v$ be the corresponding eigenfunction. If $M$ is large enough, then $v$ is close to a constant multiple of $\cos \sqrt{\lambda} x$, and thus we can normalize $v$ so that $1 \leq-\min v \leq 2$ and $1 \leq \max v \leq 2$.

Then, by Theorem 2.1, we get

$$
\sup |\nabla u| \leq \sup \left|v^{\prime}\right| \leq C
$$

where the second inequality follows from Remark 1 and the above claim.

Corollary 2.4. Let $\Omega$ and $u$ be given as in Theorem 2.1. Suppose that $N=1$ and $1=\max u \geq-\min u$. Let $k(\epsilon)$ be the smallest integer such that $2^{-k(\epsilon)} \leq \epsilon$. For integers $1 \leq k<k(\epsilon)$, let

$$
I_{k}=\left[2^{-k-1}, 2^{-k}\right] \cup\left[1-2^{-k}, 1-2^{-k-1}\right]
$$

and let $I_{k(\epsilon)}=\left[0,2^{-k(\epsilon)}\right] \cup\left[1-2^{-k(\epsilon)}, 1\right]$. Then there exists a dimensional constant $C>0$ such that

$$
\sup _{x \in I_{k}}|\nabla u| \leq C 2^{-k} \text { for } 1 \leq k \leq k(\epsilon) .
$$

Proof. Suppose $u<0$ on $\Omega(0)$ and $u>0$ on $\Omega(1)$. Denote $m=-\min u$. We claim that

$$
\max _{x \in \Omega(0)} u \leq-m+3 M_{0} \epsilon,
$$

where $M_{0}$ is the upper bound for $|\nabla u|$. To see this, let

$$
s_{0}=\min \left\{x: \min _{\Omega(x)} u=-m\right\} .
$$

By Corollary 2.3 ,

$$
|\nabla u| \leq M_{0}
$$

with a dimensional constant $M_{0}$, and thus $\max _{\Omega\left(s_{0}\right)} u \leq-m+2 M_{0} \epsilon$. Assume that $\max _{\Omega(0)} u>-m+3 M_{0} \epsilon$; then $\left\{u>-m+2 M_{0} \epsilon\right\}$ has a component $A$ such that $|A|>0$ and $A \subset\left\{x \leq s_{0}\right\}$. Let

$$
\tilde{u}=\left\{\begin{array}{lll}
u & \text { for } \quad x \in \Omega-A, \\
-m+2 M_{0} \epsilon & \text { for } \quad x \in A
\end{array}\right.
$$

then

$$
\frac{\int_{\Omega_{-}}|\nabla u|^{2}}{\int_{\Omega_{-}} u^{2}}>\frac{\int_{\Omega_{-}}|\nabla \tilde{u}|^{2}}{\int_{\Omega_{-}} \tilde{u}^{2}}
$$

and we get a contradiction. A parallel argument yields

$$
\min _{x \in \Omega(1)} u \geq 1-3 M_{0} \epsilon .
$$

Now we show (2.5) using Theorems 2.1 and 2.2. First, consider the case $k=k(\epsilon)$ on the interval $\left[0,2^{-k(\epsilon)}\right] \subset[0, \epsilon]$. Recall that $m:=-\min u \leq \max u=1$ and $u \leq-m+3 M_{0} \epsilon$ on $\Omega(0)$. Let $\psi$ be the first eigenfunction of (2.2) with $\psi^{\prime} \neq 0$ 
on $(0, b), \psi(0)>0$ and $-\min \psi=m$. Observe that $\psi$ is decreasing and thus $\psi(b)=-m$.

By Theorem 2.2. $\max \psi \geq \max u$, and thus

$$
[\min u, \max u] \subset[\min \psi, \max \psi] .
$$

Also, since $\psi$ satisfies (2.4) with $v$ replaced by $\psi$,

$$
\begin{aligned}
\psi^{\prime}(x)^{2} & =\int_{x}^{b} \frac{2(n-1)}{t} \psi^{\prime}(t)^{2}+\lambda\left(\psi(t)^{2}\right)^{\prime} d t \\
& =\int_{x}^{b} \frac{2(n-1)}{t} \psi^{\prime}(t)^{2} d t+\lambda\left(\psi(b)^{2}-\psi(x)^{2}\right) .
\end{aligned}
$$

Hence for $x \in J:=\{\psi \leq-m+C \epsilon\}$

$$
\psi^{\prime}(x)^{2} \leq \int_{x}^{b} \frac{2(n-1)}{t} \psi^{\prime}(t)^{2} d t+2 C m \lambda \epsilon .
$$

Assume that $\psi^{\prime}(x)^{2}=M \epsilon$ for the first time in $[x, b] \subset J$. Then by the above inequality

$$
M \epsilon \leq M \epsilon \cdot 2(n-1) \log \frac{b}{x}+2 C m \lambda \epsilon
$$

which yields $\log \frac{b}{x} \geq \frac{1-M^{-1} C(2 m \lambda)}{2(n-1)}$. Therefore if we let $M:=4 C m \lambda$, then we obtain $x \leq e^{-1 / 4(n-1)} b$.

Choose a sufficiently small-dimensional constant $c_{0}>0$ such that if $\epsilon \leq c_{0}$, then

$$
J=\{\psi \leq-m+C \epsilon\} \subset\left[e^{-1 / 4(n-1)} b, b\right] .
$$

It follows that if $\epsilon \leq c_{0}$, then

$$
\left|\psi^{\prime}\right| \leq C_{1} \sqrt{\epsilon}, C_{1}=\sqrt{4 C m \lambda} \text { on } J .
$$

By Theorem 2.1 and the above argument with $C=3 M_{0}$, we obtain that if $\epsilon \leq c_{0}$, then

$$
|D u| \leq M_{1} \sqrt{\epsilon} \text { in }\left\{u \leq-m+3 M_{0} \epsilon\right\}
$$

where $M_{1}=2 \sqrt{3 M_{0} m \lambda}$.

Using the improved gradient bound (2.9) instead of (2.7), (2.6) improves to

$$
u \leq-m+3 M_{1} \epsilon^{3 / 2} \text { on }\{0 \leq x \leq \epsilon\}
$$

Next we repeat the argument with the improved bound (2.10), i.e., with $\{\psi \leq$ $-m+C \epsilon\}$ replaced by $\left\{\psi \leq-m+3 M_{1} \epsilon^{3 / 2}\right\}$. It follows that for $\epsilon \leq c_{0}$

$$
|D u| \leq M_{2} \epsilon^{1 / 2+1 / 4}, u \leq-m+3 M_{2} \epsilon^{1+1 / 2+1 / 4} \text { on }\{0 \leq x \leq \epsilon\},
$$

where

$$
3 M_{2}:=6\left(6\left(3 M_{0} m \lambda\right)^{1 / 2} m \lambda\right)^{1 / 2}=6^{1+1 / 2} 3 M_{0}{ }^{1 / 4}(m \lambda)^{1 / 2+1 / 4} .
$$

Iteration of the above argument will yield that if $\epsilon<c_{0}$, then

$$
|D u| \leq 36 m \lambda \epsilon \leq C_{0} \epsilon \text { on }\{0 \leq x \leq \epsilon\},
$$

where $C_{0}$ is a dimensional constant.

For other intervals $\left[2^{-k-1}, 2^{-k}\right](k<k(\epsilon))$, a similar iteration can be applied with the first step

$$
\max _{x \in\left[0,2^{-k}\right]} u \leq-m+4 M_{0} 2^{-k}
$$


which follows from (2.6) and (2.7). Then a parallel argument with $\epsilon$ replaced by $2^{-k}$ proves (2.5) on $\left[2^{-k-1}, 2^{-k}\right]$ for $k$ such that $2^{-k} \leq c_{0}$. Note that, other than to derive (2.6), we do not use the fact that $\epsilon$ is the specific constant depending on $\Omega$.

For intervals near $x=1$ (i.e., for $\left[1-2^{-k}, 1-2^{-k-1}\right]$ ) the proof is divided into two cases. First if $\max u=1=-\min u$, then for $w:=-u\left(1-x, y_{1}, \ldots, y_{n-1}\right)$,

$$
-\min w=\max w=1, \quad w<0 \text { on }\{x=0\}, \text { and } w>0 \text { on }\{x=1\} .
$$

Therefore the argument for $w$ on intervals near $x=0$ gives the result for $u$ on intervals near $x=1$.

Secondly if $\max u=1>-\min u$, then as in the proof of Corollary 2.3 choose sufficiently large constants $a$ and $b$ such that $\lambda$ is the first nonzero eigenvalue of (2.1) and the first eigenfunction $v$ satisfies

$$
\max v=1 \text { and }-\min v \geq-\min u .
$$

Since $[\min u, \max u] \subset[\min v, \max v]$ and

$$
\min _{\Omega(1)} u \geq 1-3 M_{0} \epsilon=\max v-3 M_{0} \epsilon
$$

a similar reasoning as in the interval $\left[2^{-k-1}, 2^{-k}\right]$ yields the result for the interval $\left[1-2^{-k}, 1-2^{-k-1}\right]$ near $x=1$.

From Corollary 2.3, we obtain Corollary 2.5, which states that the first nodal set is located in the middle part of $\Omega$. Later in section 4, Corollary 2.5] and Theorem 2.1 will be used along with a new coordinate system to estimate the first nonzero eigenvalue $\lambda$. Based on the bound on $\lambda$, the width and location of the nodal set are derived again by using Theorem 2.1 .

Corollary 2.5. Let $\Omega$ and $u$ be given as in Theorem 2.1. Suppose $N=1$ and $\sup |u|=1$. Then there exist dimensional constants $c_{1}>0$ and $c_{2}>0$ such that

$$
c_{2} \leq|u| \leq 1 \text { for } x \in\left[0, c_{1}\right] \cup\left[1-c_{1}, 1\right]
$$

Proof. Without loss of generality, we may assume $\max |u|=u(\tilde{x}, \tilde{y})=1\left(\tilde{y} \in \mathbb{R}^{n-1}\right)$ and $u>0$ on $\{x=1\}$. By Corollary 2.3, there exists a dimensional constant $c_{1}>0$ such that $u(x, y)>1 / 2$ if $|x-\tilde{x}|<c_{1}$. Since the Courant nodal domain theorem [CH, p. 452] implies that $\Omega_{+}$and $\Omega_{-}$are connected, $u>1 / 2$ in $\left\{x>\tilde{x}-c_{1}\right\}$.

On the other hand, since $\int_{\Omega_{-}} u^{-}=\int_{\Omega_{+}} u^{+}$, Corollary 2.3 implies $-\min u \geq c_{2}$ for some dimensional constant $c_{2}>0$. Hence we obtain Corollary 2.5 by a similar reasoning for $u_{-}$as in $u_{+}$.

\section{ODE EIgenvalue estimates}

In this section we prove several lemmas on ODE eigenvalue estimates, which will be applied to the one-dimensional eigenfunction $\phi_{1}$ in section 4 . In particular Lemma 3.5 will be used to locate the nodal set in section 4, and Lemma 3.6 yields the bound on the width of the nodal set. The proof for Lemmas 3.1, 3.2, 3.3 and 3.4 are parallel to those of the corresponding lemmas in [J1]. The only difference in the proof is that, instead of the concavity of the height of the cross-section $h(x)$ for $n=2$, we have the concavity of $w^{1 / n-1}(x)$ by the Brunn-Minkowski inequality for the volume of the cross-section $w(x)$. 
Lemma 3.1. For $a \leq 1 / 2$,

$$
\inf _{\{\phi: \phi(a)=0\}} \frac{\int_{0}^{a} \phi^{\prime}(x)^{2} w(x) d x}{\int_{0}^{a} \phi(x)^{2} w(x) d x} \geq \frac{1}{2^{n-2} a^{2}} .
$$

Proof. The proof is the same as that of Lemma 4.2 of [J1, using the fact that $w(x) \leq 2^{n-1} w(t)$ for $0 \leq x \leq t \leq a$.

Lemma 3.2. There exists a constant $C>0$ depending on $n$ such that

$$
\inf _{\{\phi: \phi(a)=0\}} \frac{\int_{a}^{b} \phi^{\prime}(x)^{2} w(x) d x}{\int_{a}^{b} \phi(x)^{2} w(x) d x} \leq \frac{C}{(b-a)^{2}} .
$$

Proof. Take a test function $\phi(x)=x$. We need to show that

$$
\int_{0}^{1} w(x) d x \leq C \int_{0}^{1} x^{2} w(x) d x
$$

where $C$ depends on $n$. Multiply $w$ by a constant so that $\int_{0}^{1} w^{1 /(n-1)}(t) d t=1$. Due to the normalization and the concavity of $w^{1 /(n-1)}$, the arguments in the proof of Lemma 4.3 in [J1] yield that

$$
\int_{x}^{1} w^{1 /(n-1)}(t) d t \geq(1-x)^{2} \text { for } 0 \leq x \leq 1 .
$$

By Hölder inequality,

$$
\left(\int_{x}^{1} w(t) d t\right)^{1 /(n-1)}(1-x)^{(n-2) /(n-1)} \geq \int_{x}^{1} w^{1 /(n-1)}(t) d t \geq(1-x)^{2},
$$

and thus $W(x):=\int_{x}^{1} w(t) d t \geq(1-x)^{n}$. Therefore by integration by parts,

$$
\begin{aligned}
\int_{0}^{1} x^{2} w(x) d x=-\int_{0}^{1} x^{2} W^{\prime}(x) d x & =\int_{0}^{1} 2 x W(x) d x \\
& \geq \int_{0}^{1} 2 x(1-x)^{n} d x=2 /(n+1)(n+2),
\end{aligned}
$$

where the second inequality holds because $x^{2} W(x)=0$ for $x=0,1$.

On the other hand, since $w^{1 /(n-1)}(t)$ is concave with $w^{1 / n-1}(0), w^{1 / n-1}(1) \geq 0$, the graph of $w^{1 /(n-1)}(t), 0 \leq t \leq 1$, is above the triangle generated by $(0,0),(1,0)$ and $\left(t_{0}, w^{1 / n-1}\left(t_{0}\right)\right)$, where $w\left(t_{0}\right)=\max w$. It follows that

$$
\int_{0}^{1} w(t) d t \leq w\left(t_{0}\right) \leq\left(2 \int_{0}^{1} w^{1 /(n-1)}(t) d t\right)^{n-1}=2^{n-1},
$$

and our lemma holds with $C=C(n)=\frac{2^{n}}{(n+1)(n+2)}$.

Lemma 3.3. Let $s_{1}$ be the zero point of $\phi_{1}$ given in (1.4). Then there exist constants $c_{1}>0$ and $c_{2}>0$ depending on $n$ such that

$$
c_{1}<s_{1}<c_{2} \text {. }
$$

Proof. The lemma follows from Lemmas 3.1, 3.2 and the proof of Lemma 4.4 in [J1]. 
For $a \in[0,1]$, define

$$
E[a, 1]=\inf _{\{\phi: \phi(a)=0\}} \frac{\int_{a}^{1} \phi^{\prime}(x)^{2} w(x) d x}{\int_{a}^{1} \phi(x)^{2} w(x) d x} .
$$

Lemma 3.4. Suppose that $c_{0} \leq a \leq 1-c_{0}$ for some $0<c_{0}<1$. Then there exists a constant $C>0$ depending on $n$ and $c_{0}$ such that

$$
(\partial / \partial a) E[a, 1] \geq C
$$

Proof. Normalize $w$ so that

$$
\max _{0 \leq x \leq N} w(x)=1
$$

By the concavity of $w^{1 /(n-1)}$ and (3.1),$w^{1 /(n-1)}\left(c_{0}\right) \geq c_{0}$. Let $\phi$ be the unique nonnegative minimizer for $E[a, 1]$ with the normalization

$$
\int_{a}^{1} \phi(x)^{2} w(x) d x=1
$$

Following the proof of Lemma 3.4 in [J1], we only need to prove that

$$
|\phi(x)| \leq C E^{n} \text { for } a \leq x \leq 1,
$$

where $E=E[a, 1]$ and $C$ is a constant depending on $n$ and $c_{0}$.

Observe that since $\phi$ satisfies $-\left(w \phi^{\prime}\right)^{\prime}=E w \phi$ and $\phi^{\prime}(1)=0$,

$$
\begin{aligned}
\left|w \phi^{\prime}\right|(x) & \leq\left|\int_{x}^{1} E w(t) \phi(t) d t\right| \\
& \leq E\left(\int_{x}^{1} \phi^{2}(t) w(t) d t\right)^{1 / 2}\left(\int_{x}^{1} w(t) d t\right)^{1 / 2} .
\end{aligned}
$$

In particular, (3.1) and (3.2) imply that $\left|w \phi^{\prime}\right| \leq E$ for $a \leq x<1$. Since $\phi(a)=0$, we have

$$
\phi(t) \leq E \int_{a}^{t} \frac{d s}{w(s)}
$$

On the other hand, by concavity of $w^{1 / n-1}(t)$, for $a \leq s \leq t \leq 1, w^{1 / n-1}(s)$ is above the line $l(s)=\alpha s+\beta$ connecting $\left(c_{0}, c_{0}\right)$ and $\left(t, w^{1 / n-1}(t)\right)$. Without loss of generality, we may assume that $c_{0}>w^{1 / n-1}(t)$ and $\alpha<0$. (Other cases are better.) For $n \geq 3$

$$
\int_{a}^{t} \frac{d s}{w(s)} \leq \int_{c_{0}}^{t} \frac{d s}{(\alpha s+\beta)^{n-1}} \leq \frac{C}{(\alpha t+\beta)^{n-2}}=\frac{C}{w^{(n-2) / n-1}(t)}
$$


Hence by (3.4), (3.5) and (3.6)

$$
\begin{aligned}
\left|\phi^{\prime}(x)\right| & \leq \frac{E}{w(x)} \int_{x}^{1} w(t) \phi(t) d t \\
& \leq \frac{E^{2}}{w(x)} \int_{x}^{1} w(t)\left(\int_{a}^{t} \frac{1}{w(s)} d s\right) d t \\
& \leq \frac{C E^{2}}{w(x)} \int_{x}^{1} w^{1 /(n-1)}(t) d t \\
& \leq C E^{2} \frac{(1-x)}{w^{(n-2) /(n-1)}(x)} \\
& \leq C E^{2} \frac{1}{w^{(n-3) /(n-1)}(x)}
\end{aligned}
$$

where the fourth and fifth inequalities follow respectively from

$$
w^{1 /(n-1)}(t) \leq C(n) w^{1 /(n-1)}(x) \text { for } a \leq x \leq t \leq 1
$$

and

$$
w^{1 /(n-1)}(x) \geq \min \left\{1-x, c_{0}\right\} \text { for } a \leq x \leq 1 .
$$

Equations (3.8) and (3.9) are due to the concavity of $w^{1 /(n-1)}$ and the normalization (3.1). (For (3.8), see Remark 4.1 (b) in [J1.)

Equations (3.7) and (3.8) yield that

$$
|\phi(x)| \leq \int_{a}^{x}\left|\phi^{\prime}(t)\right| d t \leq \frac{C_{1} E^{2}}{w^{(n-3) /(n-1)}(x)} \text { for } a \leq x \leq 1,
$$

where $C_{1}$ is a constant depending on $n$ and $c_{0}$. We go back to the first inequality of (3.7) and apply (3.10) and then (3.8) and (3.9) to obtain

$$
\left|\phi^{\prime}(x)\right| \leq \frac{C_{1} E^{2}}{w(x)} \int_{x}^{1} w(t)^{2 /(n-1)} d t \leq \frac{C_{2} E^{3}}{w(x)^{(n-4) /(n-1)}},
$$

where $C_{2}$ is a dimensional constant. Now by similar reasoning as in (3.10), the improved estimate on $|\phi|$ holds:

$$
|\phi(x)| \leq \frac{C_{3} E^{n}}{w(x)^{(n-4) /(n-1)}} .
$$

We repeat the above process $(n-4)$ more times to obtain $|\phi(x)| \leq C(n) E^{n}$.

Lemma 3.5. Let $\phi_{1}$ and $s_{1}$ be given as in (1.4) and suppose $N=1$. If $\phi$ is a function on $(0,1)$ such that $\phi\left(s_{1}^{\prime}\right)=0$ and

$$
\frac{\int_{s_{1}^{\prime}}^{1}\left(\phi^{\prime}\right)^{2} w d s}{\int_{s_{1}^{\prime}}^{1} \phi^{2} w d s} \leq(1+M \epsilon) \frac{\int_{s_{1}}^{1}\left(\phi_{1}^{\prime}\right)^{2} w d s}{\int_{s_{1}}^{1} \phi_{1}^{2} w d s},
$$

then $s_{1}^{\prime} \leq s_{1}+C \epsilon$ for some constant $C$ depending on $n$ and $M$.

Proof. The lemma follows from Lemmas $3.3,3.4$ and from the fact

$$
E\left[s_{1}, 1\right]=\mu_{1}=\frac{\int_{s_{1}}^{1}\left(\phi_{1}^{\prime}\right)^{2} w d s}{\int_{s_{1}}^{1} \phi_{1}^{2} w d s} .
$$


Next we show that if the energy associated with $\phi$ is bounded by $(1+M \epsilon) \mu_{1}$, then $\sup |\phi|$ is bigger than $\epsilon$ on any interval of length $C \epsilon$.

Lemma 3.6. Let $N=1$ and $\mu_{1}$ be given in (1.4). Suppose $\phi(s)$ is a function on $(0,1)$ such that $\int_{0}^{1} \phi w d s=0, \sup |\phi|=1, \sup \left|\phi^{\prime}\right| \leq C_{1}$ and

$$
\mu_{1} \leq \frac{\int_{0}^{1}\left(\phi^{\prime}\right)^{2} w d s}{\int_{0}^{1} \phi^{2} w d s} \leq(1+M \epsilon) \mu_{1} .
$$

If for some $0<a<b<1$ and $C_{2}>0$

$$
\sup _{[a, b]}|\phi| \leq 2 C_{1} \epsilon \text { and }\left|\int_{a}^{1} \phi w d s\right| \geq C_{2} \int_{0}^{1} w d s,
$$

then $b-a<C \epsilon$ for some $C>0$ depending on $C_{1}, C_{2}$ and $M$.

Proof. Let $\phi_{1}$ and $s_{1}$ be as given in (1.4). Suppose that

$$
\sup _{[a, b]}|\phi| \leq 2 C_{1} \epsilon \text { and } b=a+C \epsilon,
$$

for some $a$ and sufficiently large $C>0$. Without loss of generality we may assume

$$
a \geq s_{1}+C \epsilon / 4 \text { or } b \leq s_{1}-C \epsilon / 4 .
$$

(If $a<s_{1}<b$, then $s_{1}-a \geq C \epsilon / 2$ or $b-s_{1} \geq C \epsilon / 2$. If $b-s_{1} \geq C \epsilon / 2$, replace $a$ with $\left(s_{1}+b\right) / 2$. If $s_{1}-a \geq C \epsilon / 2$, replace $b$ with $\left(s_{1}+a\right) / 2$. Lastly if $a<b<s_{1}$ or $s_{1}<a<b$, by replacing $a$ or $b$ with $(a+b) / 2$ we get (3.12).)

Changing the sign of $\phi$ if needed, we also set

$$
\left|\int_{a}^{1} \phi w d s\right|=\int_{a}^{1} \phi w d s .
$$

Define

$$
A_{\phi}=\frac{\int_{0}^{a} \phi^{2} w d s}{\int_{0}^{1} \phi^{2} w d s}, \quad B_{\phi}=\frac{\int_{a}^{1} \phi^{2} w d s}{\int_{0}^{1} \phi^{2} w d s}, A_{\phi}+B_{\phi}=1 .
$$

By our hypothesis,

$$
\frac{\int_{0}^{1}\left(\phi^{\prime}\right)^{2} w d s}{\int_{0}^{1} \phi^{2} w d s}=A_{\phi} \frac{\int_{0}^{a}\left(\phi^{\prime}\right)^{2} w d s}{\int_{0}^{a} \phi^{2} w d s}+B_{\phi} \frac{\int_{a}^{1}\left(\phi^{\prime}\right)^{2} w d s}{\int_{a}^{1} \phi^{2} w d s} \leq(1+M \epsilon) \mu_{1} .
$$

If $C$ is sufficiently large, then Lemma 3.4 and the above inequality imply that

$$
\frac{\int_{0}^{a}\left(\phi^{\prime}\right)^{2} w d s}{\int_{0}^{a} \phi^{2} w d s} \leq \mu_{1} \leq \frac{\int_{a}^{1}\left(\phi^{\prime}\right)^{2} w d s}{\int_{a}^{1} \phi^{2} w d s} .
$$

We will construct a test function $\psi$ such that $\int_{0}^{1} \psi w d s=0$ and

$$
(1+M \epsilon) \frac{\int_{0}^{1}\left(\psi^{\prime}\right)^{2} w d s}{\int_{0}^{1} \psi^{2} w d s}<\frac{\int_{0}^{1}\left(\phi^{\prime}\right)^{2} w d s}{\int_{0}^{1} \phi^{2} w d s},
$$

which contradicts our hypothesis. 
First, construct a continuous function $\tilde{\psi}$ such that $\tilde{\psi}=\phi$ on the left interval $[0, a]$ and $\tilde{\psi}=\phi+C_{2}(b-a) / 10$ on the left interval $[b, 1]$. For $\alpha=C_{2} / 10$, let

$$
\tilde{\psi}(s)=\left\{\begin{array}{lll}
\phi(s) & \text { for } \quad 0 \leq s \leq a, \\
\phi(s)+\alpha(s-a) & \text { for } \quad a \leq s \leq b=a+C \epsilon, \\
\phi(s)+\alpha(b-a) & \text { for } \quad b \leq s \leq 1 .
\end{array}\right.
$$

From a straightforward calculation, it follows that the energy of $\tilde{\psi}$ on $[a, 1]$ gets smaller than the energy of $\phi$ on $[a, 1]$ by some amount. More precisely

$$
\frac{\int_{a}^{1} \tilde{\psi}^{\prime 2} w d s}{\int_{a}^{1} \tilde{\psi}^{2} w d s} \leq\left(1-C_{0} C \epsilon\right) \frac{\int_{a}^{1} \phi^{\prime 2} w d s}{\int_{a}^{1} \phi^{2} w d s}
$$

for some $C_{0}>0$ depending on $C_{1}$ and $C_{2}$.

Next we perturb $\tilde{\psi}$ and get $\psi$ such that $\int_{0}^{1} \psi w d s=0$, and will show that $\psi$ satisfies (3.15). Set

$$
\psi(s)=\left\{\begin{array}{lll}
\tilde{\psi}(s)=\phi(s) & \text { for } & 0 \leq s \leq a, \\
\beta(\tilde{\psi}(s)-\phi(a))+\phi(a) & \text { for } & a \leq s \leq 1,
\end{array}\right.
$$

where $\beta>0$ is chosen to satisfy $\int_{0}^{1} \psi w d s=0$, i.e., $\int_{a}^{1} \psi w d s=\int_{a}^{1} \phi w d s$. Then

$$
\beta=1-\alpha|b-a| \frac{\int_{a}^{1} w d s}{\int_{a}^{1} \phi w d s}+O\left(\epsilon^{2}\right) .
$$

Since

$$
\int_{a}^{1} \phi w d s \leq \sqrt{\int_{a}^{1} \phi^{2} w d s} \sqrt{\int_{a}^{1} w d s}
$$

it follows that

$$
\int_{a}^{1} \tilde{\psi}^{2} w d s \leq\left(1+2 \alpha|b-a| \frac{\int_{a}^{1} w d s}{\int_{a}^{1} \phi w d s}+C_{3} \epsilon^{2}\right) \int_{a}^{1} \phi^{2} w d s .
$$

Therefore

$$
\int_{a}^{1} \psi^{2} w d s \leq\left(1+C_{3} \epsilon^{2}\right) \int_{a}^{1} \phi^{2} w d s
$$

and

$$
B_{\psi} \leq\left(1+C_{3} \epsilon^{2}\right) B_{\phi},
$$


where $B_{\psi}$ is defined as in (3.13) (Recall that $A_{\psi}=1-B_{\psi}$.) Therefore,

$$
\begin{aligned}
\frac{\int_{0}^{1}\left(\psi^{\prime}\right)^{2} w d s}{\int_{0}^{1} \psi^{2} w d s} & =A_{\psi} \frac{\int_{0}^{a}\left(\psi^{\prime}\right)^{2} w d s}{\int_{0}^{a} \psi^{2} w d s}+B_{\psi} \frac{\int_{a}^{1}\left(\psi^{\prime}\right)^{2} w d s}{\int_{a}^{1} \psi^{2} w d s} \\
& =A_{\psi} \frac{\int_{0}^{a}\left(\phi^{\prime}\right)^{2} w d s}{\int_{0}^{a} \phi^{2} w d s}+B_{\psi} \frac{\int_{a}^{1}\left(\psi^{\prime}\right)^{2} w d s}{\int_{a}^{1} \psi^{2} w d s} \\
& \leq\left(1+C_{3} \epsilon^{2}\right)\left(A_{\phi} \frac{\int_{0}^{a}\left(\phi^{\prime}\right)^{2} w d s}{\int_{0}^{a} \phi^{2} w d s}+B_{\phi} \frac{\int_{a}^{1}\left(\psi^{\prime}\right)^{2} w d s}{\int_{a}^{1} \psi^{2} w d s}\right) \\
& \leq\left(1+C_{3} \epsilon^{2}\right)\left(A_{\phi} \frac{\int_{0}^{a}\left(\phi^{\prime}\right)^{2} w d s}{\int_{0}^{a} \phi^{2} w d s}+B_{\phi}\left(1-C_{0} C \epsilon\right) \frac{\int_{a}^{1}\left(\phi^{\prime}\right)^{2} w d s}{\int_{a}^{1} \phi^{2} w d s}\right) \\
& \leq A_{\phi} \frac{\int_{0}^{a}\left(\phi^{\prime}\right)^{2} w d s}{\int_{0}^{a} \phi^{2} w d s}+B_{\phi}\left(1-C_{0} C \epsilon\right) \frac{\int_{a}^{1}\left(\phi^{\prime}\right)^{2} w d s}{\int_{a}^{1} \phi^{2} w d s}+C_{3} \epsilon^{2}(1+M \epsilon) \mu_{1} \\
& \leq \frac{\int_{0}^{1}\left(\phi^{\prime}\right)^{2} w d s}{\int_{0}^{1} \phi^{2} w d s}-B_{\phi} C_{0} C \epsilon \cdot \frac{\int_{a}^{1}\left(\phi^{\prime}\right)^{2} w d s}{\int_{a}^{1} \phi^{2} w d s}+C_{3} \epsilon^{2}(1+M \epsilon) \mu_{1},
\end{aligned}
$$

where we obtain the first inequality from $A_{\phi}+B_{\phi}=A_{\psi}+B_{\psi}=1$, (3.18) and (3.14), the second inequality from (3.16), and the third inequality from (3.11). From the hypothesis $\int_{a}^{1} \phi w d s \geq C_{2} \int_{0}^{1} w d s$ and (3.17), one can observe that $B_{\phi}$ is bounded below by a constant depending on $C_{2}$. Hence if we choose a sufficiently large $C$ depending on $C_{1}, C_{2}$ and $M$, and if $\epsilon$ is sufficiently small compared to $C$, then (3.15) holds.

In the next lemma, we show the nondegeneracy of $\phi_{1}$ near the zero $s_{1}$.

Lemma 3.7. Let $\phi_{1}$ be the first nonzero eigenfunction of (1.4) with $N=1$ and let $s_{1}$ be the zero of $\phi_{1}$; note that $c_{1} \leq s_{1} \leq c_{2}$ by Lemma 3.3. Normalize $\phi_{1}$ such that $\phi_{1}>0$ on $\left(s_{1}, 1\right]$ and $\max \phi_{1}=1$. Then $\phi_{1}^{\prime} \geq 0$ on $[0,1]$. Moreover there exists a dimensional constant $c=c(n)$ such that

$$
\phi_{1}^{\prime} \geq c(n) \text { on }\left[s_{1}-c_{1} / 2, s_{1}+\left(1-c_{2}\right) / 2\right] .
$$

Proof. By the normalization $\phi_{1}>0$ on $\left(s_{1}, 1\right]$ and $\phi_{1}<0$ on $\left[0, s_{1}\right)$. Hence from (1.4) we observe that $w \phi_{1}^{\prime}$ has its maximum at $x=s_{1}$ and $w \phi_{1}^{\prime}$ is increasing on $\left[0, s_{1}\right]$ and decreasing on $\left[s_{1}, 1\right]$. From the boundary condition it follows that $\phi_{1}^{\prime} \geq 0$ on $[0,1]$ and thus $\phi_{1}(1)=1$. We will only prove that $\phi_{1}^{\prime} \geq c(n)$ on $\left[s_{1}, s_{1}+\left(1-c_{2}\right) / 2\right]$ : a parallel argument leads to the rest of the claim.

Observe that from (1.4) we have

$$
\phi_{1}^{\prime}(x)=\frac{\int_{x}^{1} \mu_{1} w(t) \phi_{1}(t) d t}{w(x)} .
$$

We will use (3.19) to find lower bounds on $\phi^{\prime}$ respectively near $t=1$ and then near $t=s_{1}$, using the fact that $w \phi^{\prime}$ has its maximum at $s_{1}$. Note that for $c_{1} \leq x \leq t \leq 1$, the concavity of $w^{1 /(n-1)}(x)$ implies

$$
(1-t) w^{1 /(n-1)}(x) \leq w^{1 /(n-1)}(t) .
$$


(See Remark 4.1 (a) in [J1.) Thus (3.8) and (3.20) imply that

$$
C_{1}(1-x)^{n} w(x) \leq \int_{x}^{1} w(t) d t \leq C_{2}(1-x) w(x),
$$

where $C_{1}$ and $C_{2}$ are positive-dimensional constants. Let $\phi_{1}\left(s_{2}\right)=1 / 2$ for $s_{2} \in$ $\left[s_{1}, 1\right]$. Then by (3.19) and (3.21), it follows that for $x \in\left[s_{2}, 1\right]$,

$$
C_{1} \mu_{1}(1-x)^{n+1} \leq \phi_{1}^{\prime}(x) \leq C_{2} \mu_{1}(1-x) .
$$

Since $\phi_{1}(1)=1$, (3.22) implies that $\phi_{1}(x) \geq 1-C_{3} \mu_{1}(1-x) \geq 1 / 2$ if $x \in[A, 1]$, where $C_{3}$ and $A$ are dimensional constants. Therefore $s_{2} \leq A$ and

$$
\phi_{1}^{\prime}\left(s_{2}\right) \geq C_{1} \mu_{1}\left(1-s_{2}\right)^{n+1} \geq C_{4},
$$

where $C_{4}$ is a dimensional constant. Now we have, for $s_{1} \leq t \leq s_{2}$,

$$
\phi_{1}^{\prime}(t) \geq \frac{w\left(s_{2}\right)}{w(t)} \phi_{1}^{\prime}\left(s_{2}\right) \geq C_{5} \phi_{1}^{\prime}\left(s_{2}\right) \geq C_{6}
$$

where the second inequality is due to $t \leq s_{2}<A$ and the last from (3.23). For $t \in\left[s_{1}, s_{1}+\left(1-c_{2}\right) / 2\right]$, (3.22) implies $\phi_{1}^{\prime}(t) \geq C_{7}$, and our claim is proved.

\section{A NEW COORDinate SYSTEM}

In this section, we define a new coordinate system for $\Omega$ satisfying certain properties, which will enable us to use Fubini's Theorem when we construct a onedimensional test function with small energy. For the proof of the main lemma (Lemma 4.1), first we will prove Lemma 4.2 for general dimensions. Then we construct in detail coordinate systems for $n=3$ and $n=4$ satisfying the properties given in the main lemma. We will then discuss the general dimension based on the three- and four-dimensional cases.

Lemma 4.1. Let $\Omega$ be a bounded convex domain in $\mathbb{R}^{n}$ satisfying (1.2) and (1.3). Suppose $N=1$. Then there exists a coordinate system $\left(s, t_{1}, \ldots, t_{n-1}\right) \in[0,1] \times B_{1}(0)$ to $\left(x, y_{1}, \ldots, y_{n-1}\right) \in \Omega$ such that the following statements hold:

(I) $s=x$,

(II) $\left|\partial_{s} y_{j}\right| \leq C \epsilon \max \{1 / s, 1 /(1-s)\}$ for a dimensional constant $C$,

(III) the mapping $f:\left(s, t_{1}, \ldots, t_{n-1}\right) \rightarrow\left(s, y_{1}, \ldots, y_{n-1}\right)$ has a constant Jacobian $a_{n} w(s)$, where $w(s)$ is the volume of cross-section $\Omega(s)$ and $a_{n}$ is a dimensional constant.

Here we denote by $\partial_{s} y_{j}=\partial y_{j} / \partial s$ the partial derivative of $y_{j}$ with $t_{1}, \ldots, t_{n-1}$ held fixed. For the proof of Lemma 4.1, we need the following lemma.

Lemma 4.2. Let $\mathcal{D}$ be a planar bounded convex domain. Suppose $(0, \epsilon) \in \partial \mathcal{D}$, $\left(0,-C_{1} \epsilon\right) \in \partial \mathcal{D}\left(C_{1}>0\right)$, and $( \pm \epsilon, 0) \in \mathcal{D}$. Further suppose that the length of projection of $\mathcal{D}$ on the $y$-axis is less than $C_{2} \epsilon$ for some $C_{2}>0$. If

$$
(r \cos \theta, r \sin \theta) \in \partial \mathcal{D} \text { and }(s \cos (\theta-\Delta \theta), s \sin (\theta-\Delta \theta)) \in \partial \mathcal{D},
$$

then there exists $C$ depending on $C_{1}$ and $C_{2}$ such that

$$
|r-s| \leq C r^{2} \Delta \theta / \epsilon
$$

for sufficiently small $\Delta \theta$. 
Proof. Suppose $0 \leq \theta \leq \pi / 4$. Consider a line $l$ passing through $(0, \epsilon)$ and $(r \cos \theta$, $r \sin \theta)$. Let

$$
(\tilde{s} \cos (\theta-\Delta \theta), \tilde{s} \sin (\theta-\Delta \theta)) \in l
$$

and

$$
(s \cos (\theta-\Delta \theta), s \sin (\theta-\Delta \theta)) \in \partial \mathcal{D} .
$$

Since $\Omega$ is convex, $s \leq \tilde{s}$. The equation for $l$ is $y=\frac{r \sin \theta-\epsilon}{r \cos \theta} x+\epsilon$, and thus

$$
\tilde{s} \sin (\theta-\Delta \theta)=\frac{r \sin \theta-\epsilon}{r \cos \theta} \tilde{s} \cos (\theta-\Delta \theta)+\epsilon .
$$

If $\Delta \theta$ is sufficiently small,

$$
s \leq \tilde{s} \leq r\left(1+2\left(\frac{r \cos \theta}{\epsilon}+\frac{r \sin \theta \tan \theta}{\epsilon}\right) \Delta \theta\right) \leq r\left(1+C \frac{r \Delta \theta}{\epsilon}\right) .
$$

On the other hand, if $\tilde{l}$ is a line passing through $\left(0,-C_{1} \epsilon\right)$ and $(r \cos \theta, r \sin \theta)$, by a similar reasoning

$$
r \leq s\left(1+C \frac{s \Delta \theta}{\epsilon}\right)
$$

for $C$ depending on $C_{1}$, and the proof is done for $0 \leq \theta \leq \pi / 4$.

Next, suppose $\pi / 4 \leq \theta \leq \pi / 2$. From the hypothesis on $\Omega$, the angle between the tangent line to $\partial \Omega$ at $(r \cos \theta, r \sin \theta)$ and the line connecting the origin to $(r \cos \theta, r \sin \theta)$ is bounded below by an angle depending on $C_{2}$. Hence,

$$
|s-r| \leq C r \Delta \theta \leq C \frac{r^{2} \Delta \theta}{\epsilon}
$$

for some $C$ depending on $C_{2}$, where the second inequality follows from $r \leq C \epsilon$.

Using Lemma 4.2, we prove Lemma 4.1

Proof of Lemma 4.1. Without loss of generality, we may assume that

$$
(0,0, \ldots, 0),(1,0, \ldots, 0) \in \partial \Omega
$$

since if $l=\left\{\left(x, l_{1}(x), \ldots, l_{n-1}(x)\right): 0 \leq x \leq 1\right\}$ is a line segment connecting a point on $\bar{\Omega} \cap\{x=0\}$ to a point on $\bar{\Omega} \cap\{x=1\}$, then $1 \leq \operatorname{length}(l) \leq 1+C \epsilon$ and $\left|l_{j}^{\prime}\right| \leq C \epsilon$ for $1 \leq j \leq n-1$ and $C$ depending on $n$.

Denote $a_{i}=2^{-i}, b_{i}=1-2^{-i}\left(a_{1}=b_{1}\right)$. For $i \geq 1$, set

$$
I_{i}=\left(a_{i+1}, a_{i}\right), \quad I_{-i}=\left(b_{i}, b_{i+1}\right) .
$$

With this notation, the condition (II) of Lemma 4.1 becomes equivalent to the following statement:

$$
\left|\partial_{s} y_{j}\right| \leq C 2^{i} \epsilon \text { for } s \in I_{ \pm i}
$$

which will be verified in the proof instead of (II).

Recall that $\Omega(s)=\Omega \cap\{x=s\}$. Denote by $h_{1}(\Omega(x))$ the minimum length of projection of $\Omega(x) \subset \mathbb{R}^{n-1}$ on a line $l_{1} \subset \mathbb{R}^{n-1}$, and denote by $h_{j}(\Omega(x)), j=2, \ldots, n-1$, the minimum length of projection of $\Omega(x)$ on a line $l_{j}$ which is perpendicular to $l_{k}$ for $1 \leq k \leq j-1$ (see Figure 1). Since $\Omega$ is convex, each $\Omega_{i}:=\Omega \cap\left\{x \in I_{i}\right\}$ has orthonormal basis

$$
e_{i 1}, e_{i 2}, \ldots, e_{i n-1} \in \mathbb{R}^{n-1}
$$



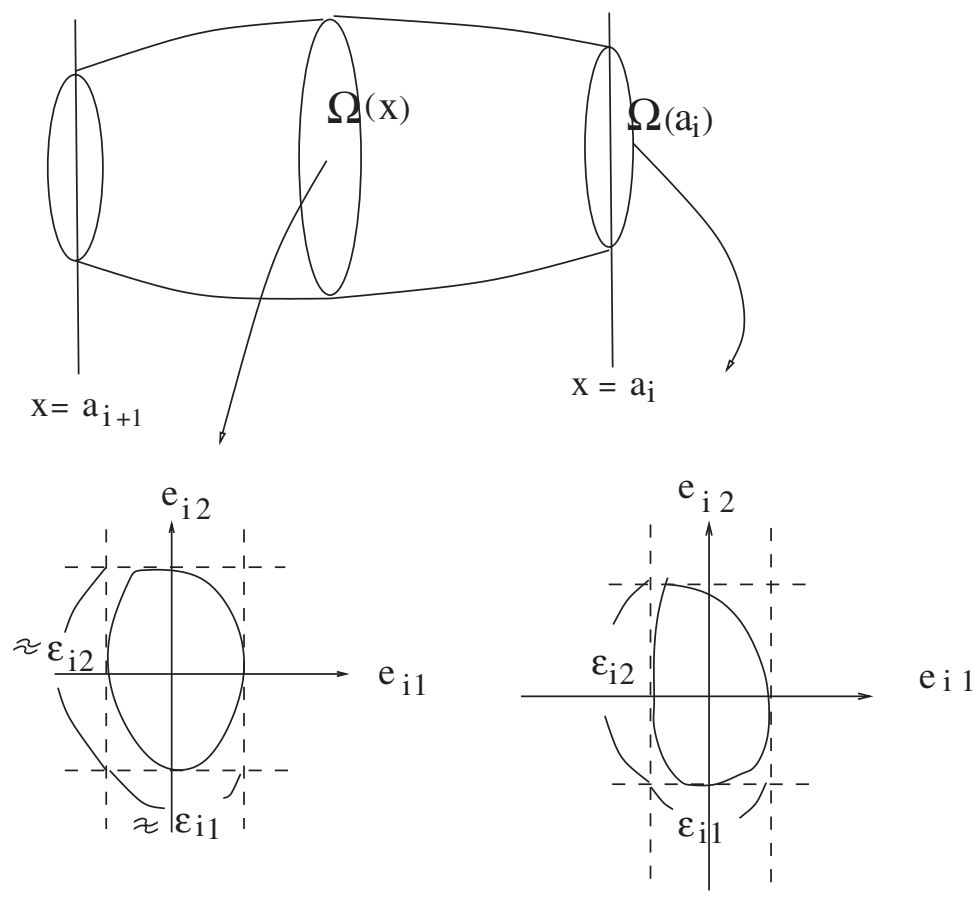

FIGURE 1

such that for $x \in I_{i}$,

$$
\left\{\begin{array}{l}
\left|p_{e_{i 1}}(\Omega(x))\right| \approx h_{1}(\Omega(x)) \approx h_{1}\left(\Omega\left(a_{i}\right)\right) \\
\cdot \\
\left|p_{e_{i n-1}}(\Omega(x))\right| \approx h_{n-1}(\Omega(x)) \approx h_{n-1}\left(\Omega\left(a_{i}\right)\right),
\end{array}\right.
$$

where $\left|p_{e_{i j}}(\Omega(x))\right|$ denotes the length of projection of $\Omega(x)$ on a line parallel to $e_{i j}$. Denote

$$
\epsilon_{i 1}=h_{1}\left(\Omega\left(a_{i}\right)\right), \ldots, \epsilon_{i n-1}=h_{n-1}\left(\Omega\left(a_{i}\right)\right),
$$

so that $\Omega(x)$ has dimensions comparable to $\epsilon_{i 1}, \ldots, \epsilon_{i n-1}$ for $x \in I_{i}$, where $\epsilon_{i 1} \leq$ $\epsilon_{i 2} \leq \ldots \leq \epsilon_{i n-1}$.

For a domain $D \in \mathbb{R}^{n}$, define the center of mass of $D$ as

$$
\frac{1}{|D|} \int_{D} x d x
$$

where $|D|$ denotes the volume of $D$. Let $(x, L(x))$ be the curve in $\Omega$ that is linear on each interval $I_{ \pm i}$ and equal to the center of mass of the cross-section at each endpoint of $I_{i}$. In other words, $L\left(a_{i}\right)$ is the center of mass of $\Omega\left(a_{i}\right)$, and similarly for $b_{i}$. On $I_{i}$, denote

$$
L(x)=L_{1}(x) e_{i 1}+\ldots+L_{n-1}(x) e_{i n-1}, \quad x \in I_{i} .
$$

Since the line segment connecting $(0,0, \ldots, 0)$ and $(1,0, \ldots, 0)$ is contained in $\Omega$, $\left(a_{i}, 0, \ldots, 0\right) \in \Omega\left(a_{i}\right)$ and $\left(a_{i+1}, 0, \ldots, 0\right) \in \Omega\left(a_{i+1}\right)$, which imply

$$
\left|L_{j}\left(a_{i}\right)-L_{j}\left(a_{i+1}\right)\right| \leq\left|L_{j}\left(a_{i}\right)\right|+\left|L_{j}\left(a_{i+1}\right)\right| \leq C \epsilon_{i j}
$$


and hence

$$
\left|L_{j}^{\prime}(x)\right| \leq C 2^{i} \epsilon_{i j} \text { for } x \in I_{i} .
$$

4.1. $n=3$. First, when $n=3$, we define a new coordinate system $\left(s, t_{1}, t_{2}\right) \in$ $[0,1] \times B_{1}(0)$ to $(x, y, z) \in \Omega$ as follows:

(i) $x=s,(y, z)=f\left(s, t_{1}, t_{2}\right)$,

(ii) $f(s, 0,0)=L(s), f(s, 1,0)-f(s, 0,0)$ is parallel to $e_{i 1}$ for every $s \in I_{i}$,

(iii) the mapping $f:\left(t_{1}, t_{2}\right) \rightarrow(y, z)$, with $s$ held fixed, is linear on every line segment from $(0,0)$ to $\partial B_{1}(0)$,

(iv) the mapping $f:\left(t_{1}, t_{2}\right) \rightarrow(y, z)$, with $s$ held fixed, has a constant Jacobian $w(s) / \pi$, where $w(s)$ is the area of $\Omega(s)$.

Note that with $s$ held fixed, $f\left(t_{1}, t_{2}\right)$ is defined so that $B_{1}(0) \cap\{0 \leq \theta \leq a\}$ is mapped onto a sector $\Omega(s) \cap\left\{0 \leq \angle\left(e_{i 1},\left(y_{1}, y_{2}\right)-L(s)\right) \leq b\right\}$ with volume $V$ satisfying

$$
\frac{a}{2 \pi}=\frac{V}{|\Omega(s)|} .
$$

(Here, $\angle\left(v_{1}, v_{2}\right)$ denotes the angle between $v_{1}$ and $v_{2}$; that is,

$$
\left.\angle\left(v_{1}, v_{2}\right)=\arccos \left(\frac{\left(v_{1}, v_{2}\right)}{\left|v_{1}\right|\left|v_{2}\right|}\right) .\right)
$$

Observe that if $g_{1}(s)$ is a function on $I_{i}$ such that

$$
\left\{\left(s, g_{1}(s) e_{i 1}\right): s \in I_{i}\right\} \subset \partial \Omega,
$$

then by the convexity of $\Omega$,

$$
\left|g_{1}^{\prime}(s)\right| \leq C\left|g_{1}(s)\right| /|s| \leq C 2^{i} \epsilon_{i 1} \text { for } s \in I_{i} .
$$

Similarly, if $\left\{\left(s, g_{2}(s) e_{i 2}\right): s \in I_{i}\right\}$ is a curve on $\partial \Omega$, then

$$
\left|g_{2}^{\prime}(s)\right| \leq C 2^{i} \epsilon_{i 2} \text { for } s \in I_{i} .
$$

Equations (4.3) and (4.4) imply that if $x \in I_{i}$ and $h>0$ is sufficiently small,

$$
|\Omega(x)-\Omega(x+h)| \leq C 2^{i} h \epsilon_{i 1} \epsilon_{i 2},
$$

where

$$
\Omega(x)-\Omega(x+h):=(\Omega(x) \cup \Omega(x+h))-(\Omega(x) \cap \Omega(x+h)) .
$$

Now to prove (II') for $x \in I_{i}$, fix $\left(t_{1}, t_{2}\right) \in \partial B_{1}(0)$. Since $\nabla L(x)$ satisfies (4.2) and $\epsilon_{i j} \leq \epsilon$, we may assume $L(x)=L(x+h)$ without loss of generality. Denote

$$
\theta_{x}=\angle\left(e_{i 1}, f\left(x, t_{1}, t_{2}\right)-L(x)\right)
$$

and

$$
r_{x}=\left|f\left(x, t_{1}, t_{2}\right)-L(x)\right| .
$$

Change the coordinates in $\mathbb{R}^{2}$ so that $L(x)=L(x+h)=0$ and

$$
f\left(x, t_{1}, t_{2}\right)=\left(r_{x} \cos \theta_{x}, r_{x} \sin \theta_{x}\right) .
$$

Fix a sufficiently small $h>0$, and denote

$$
f\left(x+h, t_{1}, t_{2}\right)=\left(r_{x+h} \cos \theta_{x+h}, r_{x+h} \sin \theta_{x+h}\right) .
$$

Since $f$ has a constant Jacobian, (4.5) implies

$$
r_{x}^{2}\left|\theta_{x+h}-\theta_{x}\right| \leq C 2^{i} h \epsilon_{i 1} \epsilon_{i 2}
$$


since

$$
\begin{aligned}
\left|\Omega(x) \cap\left\{0 \leq \theta \leq \theta_{x}\right\}-\Omega(x+h) \cap\left\{0 \leq \theta \leq \theta_{x}\right\}\right| & \leq|\Omega(x)-\Omega(x+h)| \\
& \leq C 2^{i} h \epsilon_{i 1} \epsilon_{i 2} .
\end{aligned}
$$

Let $s>0$ be a number such that $\left(s \cos \theta_{x+h}, s \sin \theta_{x+h}\right) \in \partial \Omega(x)$. Then Lemma 4.2 implies

$$
\left|s-r_{x}\right| \leq C r_{x}^{2}\left|\theta_{x+h}-\theta_{x}\right| / \epsilon_{i 1}
$$

which yields

$$
\begin{aligned}
\left|\left(r_{x} \cos \theta_{x}, r_{x} \sin \theta_{x}\right)-\left(s \cos \theta_{x+h}, s \sin \theta_{x+h}\right)\right| & \leq C r_{x}\left|\theta_{x+h}-\theta_{x}\right|+\left|s-r_{x}\right| \\
& \leq C 2^{i} h \epsilon_{i 2},
\end{aligned}
$$

where the last inequality follows from (4.6), (4.7) and $\epsilon_{i 1} \leq C r_{x}$.

On the other hand, since

$$
A:=\left(s \cos \theta_{x+h}, s \sin \theta_{x+h}\right) \text { and } B:=\left(r_{x+h} \cos \theta_{x+h}, r_{x+h} \sin \theta_{x+h}\right)
$$

are points on $\partial \Omega(x)$ and $\partial \Omega(x+h)$ with the same angle from $e_{i 1}$, a similar argument as in (4.3) and (4.4) yields

$$
|A-B| \leq C 2^{i} h s \leq C 2^{i} h \epsilon_{i 2} .
$$

Hence we conclude

$$
\begin{aligned}
\mid f\left(x, t_{1}, t_{2}\right) & -f\left(x+h, t_{1}, t_{2}\right) \mid \\
= & \left|\left(r_{x} \cos \theta_{x}, r_{x} \sin \theta_{x}\right)-\left(r_{x+h} \cos \theta_{x+h}, r_{x+h} \sin \theta_{x+h}\right)\right| \\
\leq & C 2^{i} h \epsilon_{i 2} \leq C 2^{i} h \epsilon,
\end{aligned}
$$

and this implies (II') by sending $h$ to 0 .

4.2. $n=4$. Next we consider $n=4$. Recall that for $x \in I_{i}, p_{e_{i j}}(\Omega(x)) \approx \epsilon_{i j}$ with $\epsilon_{i 1} \leq \epsilon_{i 2} \leq \epsilon_{i 3}$, and

$$
(x, L(x))=\left(x, L_{1}(x) e_{i 1}+L_{2}(x) e_{i 2}+L_{3}(x) e_{i 3}\right)
$$

is a line segment connecting the center of mass of $\Omega\left(a_{i}\right)$ to that of $\Omega\left(a_{i+1}\right)$ on $I_{i}$. To define a new coordinate system, we first divide $\Omega(x)$ into two parts with the same volume as follows. Let $\tilde{L}_{3}(x)$ be a number such that

$$
\left|\left\{y_{1} e_{i 1}+y_{2} e_{i 2}+y_{3} e_{i 3} \in \Omega(x): y_{3} \geq \tilde{L}_{3}(x)\right\}\right|=|\Omega(x)| / 2 .
$$

Let $\left(r, \theta_{1}, \theta_{2}\right)$ be a polar coordinate in $\Omega(x)$ with $r=0$ at

$$
\tilde{L}(x):=L_{1}(x) e_{i 1}+L_{2}(x) e_{i 2}+\tilde{L}_{3}(x) e_{i 3} .
$$

Let $g_{x}$ be a density function on $\partial \Omega(x)$ with total mass 1 , i.e., for any $0 \leq a_{1}, a_{2}, b_{1}, b_{2} \leq 2 \pi$

$$
\begin{array}{r}
\left|\left\{\left(r, \theta_{1}, \theta_{2}\right) \in \Omega(x): a_{1} \leq \theta_{1} \leq b_{1}, a_{2} \leq \theta_{2} \leq b_{2}\right\}\right| \\
=|\Omega(x)| \int_{\partial \Omega(x) \cap\left\{a_{j} \leq \theta_{j} \leq b_{j}, j=1,2\right\}} g_{x} d \theta_{1} d \theta_{2} .
\end{array}
$$

(Note that

$$
g_{x}(y)=C \frac{r(y)^{3}}{|\Omega(x)|}, \quad r(y)=|y-\tilde{L}(x)|
$$

where $C>0$ is a dimensional constant.) 
Now we construct a coordinate system $\left(s, t_{1}, t_{2}, t_{3}\right) \in[0,1] \times B_{1}(0)$ to $\left(x, y_{1}, y_{2}, y_{3}\right)$ $\in \Omega$ as follows:

(i) $x=s,\left(y_{1}, y_{2}, y_{3}\right)=f\left(s, t_{1}, t_{2}, t_{3}\right)$,

(ii) $f(s, 0,0,0)=\tilde{L}(s), f(s, 1,0,0)-f(s, 0,0,0)$ is parallel to $e_{i 1}$ for every $s \in I_{i}$,

(iii) the mapping $f:\left(t_{1}, t_{2}, t_{3}\right) \rightarrow\left(y_{1}, y_{2}, y_{3}\right)$, with $s$ held fixed, is linear on every line segment from $(0,0,0)$ to $\partial B_{1}(0)$,

(iv) the mapping $f:\left(t_{1}, t_{2}, t_{3}\right) \rightarrow\left(y_{1}, y_{2}, y_{3}\right)$ satisfies the following properties:

(a) $f$ maps the half sphere

$$
B_{1}^{\text {right }}(0):=\left\{\left(t_{1}, t_{2}, t_{3}\right) \in B_{1}(0): t_{3} \geq 0\right\}
$$

onto

$$
\Omega^{\text {right }}(x):=\left\{y_{1} e_{i 1}+y_{2} e_{i 2}+y_{3} e_{i 3} \in \Omega(x): y_{3} \geq \tilde{L}_{3}(x)\right\} .
$$

(b) $f$ maps the half cone

$$
B(\theta):=\left\{t \in B_{1}^{\text {right }}(0): \angle\left(t, e_{1}\right) \leq \theta\right\}
$$

onto

$$
\Omega(x, \phi(x, \theta)):=\left\{y \in \Omega^{r i g h t}(x): \angle\left(\vec{y}, e_{i 1}\right) \leq \phi(x, \theta)\right\},
$$

where $\vec{y}$ is a vector from $\tilde{L}(x)$ to $y$, and $\phi(x, \theta)$ is the angle such that

$$
\frac{|B(\theta)|}{\left|B_{1}^{\text {right }}(0)\right|}=\frac{|\Omega(x, \phi(x, \theta))|}{\left|\Omega^{\text {right }}(x)\right|} \text {. }
$$

(c) Let $\tilde{B}(\theta)=\left\{t \in \partial B_{1}^{\text {right }}(0): \angle\left(t, e_{1}\right)=\theta\right\}$ and

$$
\tilde{\Omega}(x, \phi(x, \theta))=\left\{y \in \partial \Omega^{\text {right }}(x): \angle\left(\vec{y}, e_{i 1}\right)=\phi(x, \theta)\right\} .
$$

Then the mapping $f: \tilde{B}(\theta) \rightarrow \tilde{\Omega}(x, \phi(x, \theta))$, with $s$ and $\theta$ held fixed, satisfies

$$
\frac{\int_{f(A)} g_{x}}{\int_{\tilde{\Omega}(x, \phi(x, \theta))} g_{x}}=\frac{|A|}{|\tilde{B}(\theta)|}
$$

for any $A \subset \tilde{B}(\theta)$.

(d) $f$ satisfies parallel properties for $B^{\text {left }}(0):=B_{1}(0)-B_{1}^{\text {right }}(0)$ and $\Omega^{\text {left }}(x):=\Omega(x)-\Omega^{\text {right }}(x)$.

( $f$ might be discontinuous on the intersection of $B^{\text {right }}(0)$ and $B^{\text {left }}(0)$.)

The conditions (i)-(iii) are parallel to those when $n=3$, and the only difference between $n=3$ and $n=4$ is the condition (iv). In fact, when $n=3$, there is a unique map $f:\left(s, t_{1}, t_{2}\right) \rightarrow(y, z)$ with a constant Jacobian on each cross section, if we fix one direction $e_{i 1}$ (see condition (ii)). However when $n=4$, there are infinitely many maps $f:\left(s, t_{1}, t_{2}, t_{3}\right) \rightarrow\left(y_{1}, y_{2}, y_{3}\right)$ with a constant Jacobian, even if we fix any two directions. In other words when $n=4$, the properties stated for the three-dimensional case do not suffice to construct a function $f$ which satisfies $\left(\mathrm{II}^{\prime}\right)$. Hence when $n=4$, we construct a (unique) map $f$ with a constant Jacobian, under the constraint (iv) that $f$ maps a two-dimensional surface in $B_{1}(0)$ with a fixed angle $\theta$ from $e_{1}$ to a two dimensional surface in $\Omega(x)$ with a fixed angle $\phi(x, \theta)$ from $e_{i 1}$. Then the map $f$, with $\theta$ fixed, is two-dimensional and area-preserving with respect to the normalized density function $g_{x} /\left|\int g_{x}\right|$. Hence we can proceed 


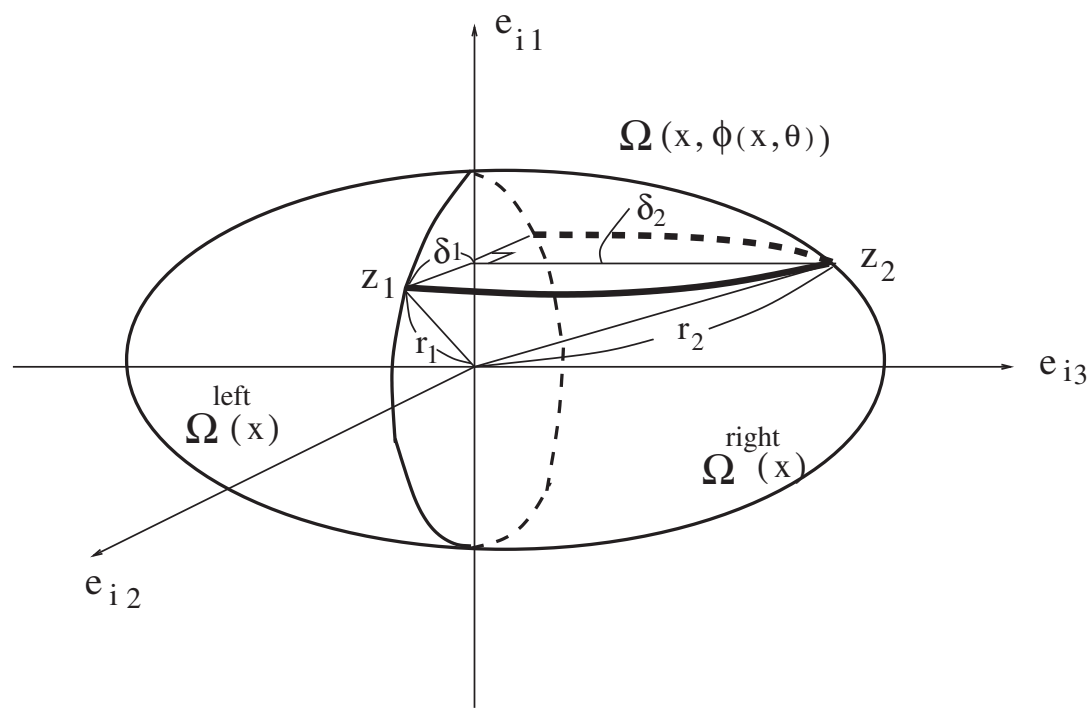

Figure 2

as in the case $n=3$. Here we divide $\Omega$ into two parts $\Omega^{\text {right }}(x)$ and $\Omega^{\text {left }}(x)$, so that the shorter arcs of

$$
\left\{y \in \Omega(x): \angle\left(y, e_{i 1}\right)=\phi(x, \theta)\right\}
$$

are mapped to the shorter arcs of

$$
\left\{y \in \Omega(\tilde{x}): \angle\left(y, e_{i 1}\right)=\phi(\tilde{x}, \theta)\right\}
$$

by $f_{\tilde{x}} \circ f_{x}^{-1}$, where $f_{x}$ and $f_{\tilde{x}}$ denote the map $f$ with $s=x$ and $s=\tilde{x}$, respectively.

Now to prove (II'), let $x \in I_{i}$ and fix a sufficiently small $h>0$. Translate $\Omega$ so that $(x, \tilde{L}(x))=0$. Let $z_{1}=a_{1} e_{i 1}+a_{2} e_{i 2}$ and $z_{2}=b_{1} e_{i 1}+b_{2} e_{i 3}$ be points on

$$
\tilde{\Omega}(x, \phi(x, \theta))
$$

(see Figure 2).

In other words, $z_{1} \in \partial \Omega(x)$ is a point on the $e_{i 1} e_{i 2}$ plane such that the angle between $e_{i 1}$ and $z_{1}$ equals $\phi(x, \theta)$. Similarly, $z_{2} \in \partial \Omega(x)$ is the point on the $e_{i 1} e_{i 3}$ plane such that the angle between $e_{i 1}$ and $z_{2}$ equals $\phi(x, \theta)$. Denote by $\delta_{1}$ the distance from $z_{1}$ to $e_{i 1}$-axis and by $\delta_{2}$ the distance from $z_{2}$ to $e_{i 1}$-axis. Since $\epsilon_{i 1} \leq \epsilon_{i 2} \leq \epsilon_{i 3}$, one can observe that $\delta_{1} \leq C \delta_{2}$ for a dimensional constant $C$. Also denote $r_{1}=\left|z_{1}\right|$ and $r_{2}=\left|z_{2}\right|$; then $r_{1} \leq C r_{2}$. Observe that since $\Omega(x, \phi(x, \theta))$ is convex,

$$
\epsilon_{i 1} \delta_{1} \delta_{2} / C \leq|\Omega(x, \phi(x, \theta))| \leq C \epsilon_{i 1} \delta_{1} \delta_{2}
$$

for a dimensional constant $C$.

On the other hand, by similar arguments as in (4.3) and (4.4)

$$
|\Omega(x)-\Omega(x+h)| \leq C 2^{i} h|\Omega(x)|
$$

for $x \in I_{i}$ and a small $h>0$. This implies

$$
\epsilon_{i 1} \epsilon_{i 2}\left|\tilde{L}_{3}(x)-\tilde{L}_{3}(x+h)\right| \leq C 2^{i} h|\Omega(x)|
$$


and thus

$$
\left|\tilde{L}_{3}^{\prime}(x)\right| \leq C 2^{i} \epsilon_{i 3}, \quad\left|L_{1}^{\prime}(x)\right| \leq C 2^{i} \epsilon_{i 1}, \quad\left|L_{2}^{\prime}(x)\right| \leq C 2^{i} \epsilon_{i 2},
$$

where the second and third inequalities follow by the same argument as in (4.2). Recall that by definition of $\phi(x, \theta)$ and $\phi(x+h, \theta)$,

$$
\frac{|\Omega(x, \phi(x, \theta))|}{|\Omega(x)|}=\frac{|B(\theta)|}{\left|B_{1}(0)\right|}=\frac{|\Omega(x+h, \phi(x+h, \theta))|}{|\Omega(x+h)|} .
$$

The above equality and (4.12) imply

$$
|| \Omega(x, \phi(x, \theta))|-| \Omega(x+h, \phi(x+h, \theta))|| \leq C 2^{i} h|\Omega(x, \phi(x, \theta))| .
$$

To simplify notation, denote

$$
\Omega_{0}=\Omega(x, \phi(x, \theta)), \quad \Omega_{2}=\Omega(x+h, \phi(x+h, \theta))
$$

and let $\Omega_{1}$ be an intermediate region such that

$$
\Omega_{1}=\Omega^{r i g h t}(x) \cap\left\{y: \angle\left(\vec{y}, e_{i 1}\right) \leq \phi(x+h, \theta)\right\} .
$$

(Note that $\Omega_{1}$ is an intermediate region in the sense that $\Omega_{0}$ and $\Omega_{1}$ are contained in $\Omega(x)$, but the angle from $\epsilon_{i 1}$ to $\partial \Omega_{1}$ is the same as the angle from $\epsilon_{i 1}$ to $\partial \Omega_{2}$.) Then by similar reasoning as in (4.3) and (4.4) and by (4.13),

$$
\left|\Omega_{1}-\Omega_{2}\right| \leq C 2^{i} h\left|\Omega_{1}\right| \leq C 2^{i} h\left|\Omega_{0}\right|
$$

if $h$ is sufficiently small so that $|\phi(x, \theta)-\phi(x+h, \theta)|$ is small enough. Hence

$$
\begin{aligned}
\left|\Omega_{0}-\Omega_{1}\right|=|| \Omega_{0}|-| \Omega_{1}|| & \leq|| \Omega_{0}|-| \Omega_{2}||+|| \Omega_{1}|-| \Omega_{2}|| \\
& \leq|| \Omega_{0}|-| \Omega_{2}||+\left|\Omega_{1}-\Omega_{2}\right| \\
& \leq C 2^{i} h\left|\Omega_{0}\right| \\
& \leq C 2^{i} h \epsilon_{i 1} \delta_{1} \delta_{2},
\end{aligned}
$$

where the first inequality is due to the fact that either $\Omega_{0} \subset \Omega_{1}$ or $\Omega_{1} \subset \Omega_{0}$, the third inequality follows from (4.14), and the last equality follows from (4.11). Denote

$$
\Delta \phi=|\phi(x+h, \theta)-\phi(x, \theta)|
$$

then by the above inequality

$$
|\Delta \phi| r_{2}^{2} \delta_{1} \leq\left|\Omega_{0}-\Omega_{1}\right| \leq C 2^{i} h \epsilon_{i 1} \delta_{1} \delta_{2},
$$

which yields

$$
|\Delta \phi| \leq C 2^{i} h \epsilon_{i 1} \delta_{2} / r_{2}^{2} .
$$

Fix $t=\left(t_{1}, t_{2}, t_{3}\right) \in \partial B_{1}(0)$. Without loss of generality, we may assume $t_{2}, t_{3} \geq$ 0 . Denote by $z$ and $w$ the points on $\Omega(x)$ and $\Omega(x+h)$ such that

$$
z=f(x, t), \quad w=f(x+h, t) .
$$

(Note that $\angle\left(e_{i 1}, \vec{z}\right)=\phi(x, \theta)$ and $\angle\left(e_{i 1}, \vec{w}\right)=\phi(x+h, \theta)$ for the angle $\theta$ between $e_{1}$ and $t$.) For the proof of $\left(\mathrm{II}^{\prime}\right)$, it suffices to show

$$
|z-w| \leq C 2^{i} h \epsilon_{i 3} \leq C 2^{i} h \epsilon .
$$

To prove (4.16), introduce intermediate points $z_{1}$ and $z_{2}$ on $\partial \Omega(x)$ such that

$$
\angle\left(e_{i 1}, \overrightarrow{z_{1}}\right)=\angle\left(e_{i 1}, \vec{z}\right), \quad \angle\left(e_{i 2}, \overrightarrow{p\left(z_{1}\right)}\right)=\angle\left(e_{i 2}, \overrightarrow{p(w)}\right)
$$

and

$$
\angle\left(e_{i 2}, \overrightarrow{p\left(z_{2}\right)}\right)=\angle\left(e_{i 2}, \overrightarrow{p\left(z_{1}\right)}\right), \quad \angle\left(e_{i 1}, \overrightarrow{z_{2}}\right)=\angle\left(e_{i 1}, \vec{w}\right)
$$


where $p$ is a projection on the $e_{i 2} e_{i 3}$-plane. Using the bound (4.15) on $\Delta \phi$, Lemma 4.2 implies

$$
\begin{aligned}
\left|z_{1}-z_{2}\right| & \leq C\left(r \Delta \phi+r^{2} \Delta \phi / \epsilon_{i 1}\right) \\
& \leq C 2^{i} h \delta_{2} r^{2} / r_{2}^{2} \\
& \leq C 2^{i} h \epsilon_{i 3}
\end{aligned}
$$

where $r=\left|z_{1}-\tilde{L}(x)\right|$, the second inequality follows from $\epsilon_{i 1} \leq C r$ and the last inequality follows from $r \leq C r_{2}$ and $\delta_{2} \leq C \epsilon_{i 3}$. Moreover, since $z_{2}$ and $w$ are points on $\Omega(x)$ and $\Omega(x+h)$ with the same angles from $e_{i 1}$ and $e_{i 2}$, (4.13) and a similar argument as in (4.3) yield that

$$
\left|z_{2}-w\right| \leq C 2^{i} h \epsilon_{i 3} .
$$

Hence it suffices to prove $|z-\tilde{z}| \leq C 2^{i} h \epsilon_{i 3}$ for the proof of (4.16).

To simplify notation, denote

$$
\theta_{2}(z)=\angle\left(e_{i 2}, \overrightarrow{p(z)}\right)
$$

where $p$ is a projection on $e_{i 2} e_{i 3}$-plane. Also let

$$
\begin{aligned}
S_{0} & =\left\{y \in \partial \Omega(x): \angle\left(e_{i 1}, y\right)=\phi(x, \theta), 0 \leq \theta_{2}(y) \leq \pi\right\}, \\
S_{1} & =\left\{y \in \partial \Omega(x): \angle\left(e_{i 1}, y\right)=\phi(x, \theta), 0 \leq \theta_{2}(y) \leq \theta_{2}(z)\right\}, \\
S_{0}^{h} & =\left\{y \in \partial \Omega(x+h): \angle\left(e_{i 1}, y\right)=\phi(x+h, \theta), 0 \leq \theta_{2}(y) \leq \pi\right\}, \\
S_{1}^{h} & =\left\{y \in \partial \Omega(x+h): \angle\left(e_{i 1}, y\right)=\phi(x+h, \theta), 0 \leq \theta_{2}(y) \leq \theta_{2}(w)\right\} .
\end{aligned}
$$

Then (4.10) implies

$$
\frac{\int_{S_{1}} g_{x}}{\int_{S_{0}} g_{x}}=\frac{\int_{S_{1}^{h}} g_{x+h}}{\int_{S_{0}^{h}} g_{x+h}} .
$$

We claim that the denominators in (4.18) are very close to each other, more precisely,

$$
\left|\int_{S_{0}} g_{x}-\int_{S_{0}^{h}} g_{x+h}\right| \leq C 2^{i} h \int_{S_{0}^{h}} g_{x} .
$$

To prove the claim, recall that

$$
g_{x}(y)=C r(y)^{3} /|\Omega(x)|,
$$

where $r(y)=|y-\tilde{L}(x)|$ and $C$ is a dimensional constant. If $y \in S_{0}$ and $y^{\prime} \in S_{0}^{h}$ are points satisfy $\theta_{2}(y)=\theta_{2}\left(y^{\prime}\right)$, then

$$
\begin{aligned}
\left|g_{x}(y)-g_{x+h}\left(y^{\prime}\right)\right| & \leq\left|\frac{C r(y)^{3}}{|\Omega(x)|}-\frac{C r(\tilde{y})^{3}}{|\Omega(x)|}\right|+C 2^{i} h g_{x}(y) \\
& =\left(\left|1-\frac{r(\tilde{y})^{3}}{r(y)^{3}}\right|+C 2^{i} h\right) g_{x}(y) \\
& \leq\left(\frac{C 2^{i} h \delta_{2} r(y)^{2}}{r_{2}^{2} r(y)}+C 2^{i} h\right) g_{x}(y) \\
& \leq C 2^{i} h g_{x}(y)
\end{aligned}
$$


where the first inequality follows from (4.12), the second inequality follows from a similar argument as in the second inequality of (4.17), and the last inequality follows from $\delta_{2} r(y) \leq r_{2}^{2}$. Thus

$$
\left|\int_{S_{0}} g_{x}-\int_{S_{0}^{h}} g_{x+h}\right| \leq C 2^{i} h \int_{S_{0}^{h}} g_{x}
$$

and due to (4.18)

$$
\begin{aligned}
\left|\int_{S_{1}} g_{x}-\int_{S_{1}^{h}} g_{x+h}\right| & \leq C 2^{i} h \int_{S_{1}} g_{x} \\
& =C 2^{i} h \int_{S_{1}} \frac{r(y)^{3}}{|\Omega(x)|} \\
& \leq C 2^{i} h r(z) \int_{S_{1}} \frac{r(y)^{2}}{|\Omega(x)|} \\
& \leq \frac{C 2^{i} h r(z)^{2} r_{1}}{|\Omega(x)|}=\frac{C 2^{i} h r^{2} r_{1}}{|\Omega(x)|}
\end{aligned}
$$

where $r=r(z)=|z-\tilde{L}(x)|$. Here the second inequality is from the fact $r(y) \leq$ $\operatorname{Cr}(z)$ on $S_{1}$ (see Figure 2). Thus

$$
\frac{r^{3}\left|\theta_{2}(z)-\theta_{2}(w)\right|}{|\Omega(x)|} \leq C\left|\int_{S_{1}} g_{x}-\int_{S_{1}^{h}} g_{x+h}\right| \leq \frac{C 2^{i} h r^{2} r_{1}}{|\Omega(x)|}
$$

which yields

$$
\Delta \theta_{2}:=\left|\theta_{2}(z)-\theta_{2}(w)\right| \leq C 2^{i} h r_{1} / r
$$

By applying Lemma 4.2 on $\partial \Omega(x, \phi(x, \theta))$ with $\epsilon=r_{1}$, we get

$$
\begin{aligned}
\left|z-z_{1}\right| & \leq C\left(r \Delta \theta_{2}+r^{2} \Delta \theta_{2} / r_{1}\right) \\
& \leq C 2^{i} h r \\
& \leq C 2^{i} h \epsilon_{i 3} .
\end{aligned}
$$

Combining (4.22) with the bounds on $\left|z_{1}-z_{2}\right|$ and $\left|z_{2}-w\right|$, (4.16) is proved.

Remark. Observe that in the construction of $f$ we divided $\Omega(x)$ into $\Omega^{\text {right }}(x)$ and $\Omega^{\text {left }}(x)$ in a way that any point $y$ on

$$
\partial \Omega(x) \cap \partial \Omega^{\text {right }}(x) \cap \partial \Omega^{l e f t}(x)
$$

has the shortest distance from the center $\tilde{L}(x)$ among the points on

$$
\left\{z \in \partial \Omega(x): \angle\left(e_{i 1}, \vec{z}\right)=\angle\left(e_{i 1}, \vec{y}\right)\right\} .
$$

In fact, if the shorter arcs were not fixed by $f$, then our bound in (4.21) would be $C 2^{i} h \tilde{r} / r$ for some $\tilde{r} \geq r_{1}$, which is not strong enough to obtain the second inequality of (4.22).

4.3. $n>4$. As in $n=4$, in each $I_{i}$ we fix an orthonormal basis $e_{i 1}, \ldots, e_{i n-1}$ as before (with $\left.\epsilon_{i 1} \leq \ldots \leq \epsilon_{i n-1}\right)$ and construct a one-to-one map $f\left(s, t_{1}, \ldots, t_{n-1}\right)$ with a constant Jacobian, under the constraint

$$
f(s, \cdot): B_{1}(0) \cap\left\{t_{n-1} \geq 0\right\} \rightarrow \Omega(x) \cap\left\{y_{n-1} \geq \tilde{L}_{n-1}(x)\right\},
$$

and $f(s, \cdot)$ maps the $(n-2)$-dimensional surface

$$
\left\{t \in B_{1}(0): \angle\left(t, e_{k}\right)=\phi_{k}\left(y, \theta_{k}\right)\right\}
$$


to

$$
\left\{y \in \Omega(x): \angle\left(x, e_{i k}\right)=\phi_{k}\left(x, \theta_{k}\right)\right\} .
$$

Then with $\theta_{1}, \ldots, \theta_{n-3}$ fixed, $f$ is a one-to-one map between two-dimensional surfaces, with shorter arcs (of length $r_{1}$ ) being fixed, and area-preserving with respect to the normalized density function $\frac{g_{x}}{\left|\int g_{x}\right|}$, where $g_{x}$ is a density function on $\partial \Omega(x) \subset R^{n-1}$ defined similarly as in (53).

Then parallel arguments as in $n=4$ would yield the corresponding inequality to (61):

$$
\left|\Delta \phi_{k}\right| \leq C 2^{i} h \epsilon_{i 1} \delta_{n-2} / r_{n-2}^{2}
$$

where

$$
\Delta \phi_{k}=\phi_{k}\left(x, \theta_{k}\right)-\phi\left(x+h, \theta_{k}\right) \text { and } 1 \leq k \leq n-3 .
$$

Thus we get (4.17) with $\tilde{z}^{\prime}$ replaced by $\tilde{z}_{k}^{\prime}$ and $\epsilon_{i 3}$ replaced by $\epsilon_{i n-1}$, where

$$
\angle\left(\tilde{z}, e_{i k}\right)=\phi_{k}\left(x, \theta_{k}\right), \quad \angle\left({\tilde{z_{k}}}^{\prime}, e_{i k}\right)=\phi_{k}\left(x+h, \theta_{k}\right)
$$

and $1 \leq k \leq n-3$.

Similar arguments as in (4.20) would yield that

$$
\frac{r^{n-1}\left|\theta_{n-2}(z)-\theta_{n-2}(w)\right|}{|\Omega(x)|} \leq C\left|\int_{S_{1}} g_{x}-\int_{S_{1}^{h}} g_{x+h}\right| \leq \frac{C 2^{i} h r^{n-2} r_{1}}{|\Omega(x)|}
$$

which would yield (4.21) and thus (4.22) with $\epsilon_{i 3}$ replaced by $\epsilon_{i n-1}$. Combining these inequalities as in $n=4$, we obtain $\left(\mathrm{II}^{\prime}\right)$.

\section{Proof of Theorem 1.1}

Throughout the proof, all dimensional constants will be denoted by $C$. We start with assuming that $\Omega$ has a smooth boundary - we will consider the general case at the end of the proof. Normalize $u$ and $\Omega$ so that $0<-\min u \leq \max u=1$ and $N=1$. In the first part of the proof, the difference between the first eigenvalues $\lambda$ and $\mu$ of (1.1) and (1.4) will be estimated by a bound $C \epsilon$. From this bound, the nodal set will be located in an $x$-interval of length $C \epsilon$, which is also near the zero $s_{1}$ of the first eigenfunction $\phi_{1}$ of the corresponding ordinary differential equation (1.4).

Let $\left(s, t_{1}, \ldots, t_{n-1}\right)$ be the new coordinate system constructed in Lemma 4.1. Using Fubini's Theorem with this new coordinate system, we can choose $\left(\tilde{t}_{1}, \ldots, \tilde{t}_{n-1}\right)$ such that

$$
\begin{aligned}
\int_{\Omega}|\nabla u|^{2} & =\int_{[0,1] \times B_{1}(0)}|\nabla u|^{2} a_{n} w(s) d s \ldots d t_{n-1} \\
& \geq \int_{0}^{1}\left|\nabla u\left(s, f\left(s, \tilde{t}_{1}, \ldots, \tilde{t}_{n-1}\right)\right)\right|^{2} w(s) d s .
\end{aligned}
$$

Based on (5.1), we will construct a one-dimensional test function $\psi$ to compare with $\phi_{1}$ as follows. Let $k_{0}$ be the smallest integer such that $2^{-k_{0}} \leq \epsilon$ and let

$$
J_{i}=\left[2^{-i-1}, 2^{-i}\right] \cup\left[1-2^{-i}, 1-2^{-i-1}\right] \text { for } 1 \leq i \leq k_{0} .
$$

Define a function $\tilde{\phi}$ in $\Omega$ such that

(i) $\tilde{\phi}(z)=\tilde{\phi}(w)$ for $z, w \in \Omega(x), 0 \leq x \leq 1$,

(ii) $\tilde{\phi}=u$ on $\left\{\left(s, f\left(s, \tilde{t}_{1}, \ldots, \tilde{t}_{n-1}\right)\right): 2^{-k_{0}} \leq s \leq 1-2^{-k_{0}}\right\}$, 
(iii) $\tilde{\phi}=\tilde{\phi}\left(2^{-k_{0}}, f\left(2^{-k_{0}}, \tilde{t}_{1}, \ldots, \tilde{t}_{n-1}\right)\right)$ for $0 \leq x \leq 2^{-k_{0}}$,

(iv) $\tilde{\phi}=\tilde{\phi}\left(1-2^{-k_{0}}, f\left(1-2^{-k_{0}}, \tilde{t}_{1}, \ldots, \tilde{t}_{n-1}\right)\right)$ for $1-2^{-k_{0}} \leq x \leq 1$.

Observe that $\tilde{\phi}$ is continuous on $J_{i}$ and may have a jump discontinuity at endpoints of $J_{i}$. But since

$$
|\nabla u| \leq C 2^{-i} \text { on } J_{i}
$$

due to Corollary 2.4 and since $\Omega(x)$ has a diameter less than $2 \epsilon$,

$$
u(z)-u(w) \leq C 2^{-i} \epsilon \text { for } z, w \in \Omega(x) .
$$

Equation (5.2) implies that there exists a continuous function $\phi$ such that for $1 \leq$ $i \leq k_{0}$

$$
\phi=\tilde{\phi}+d_{i} \text { on }\left[2^{-i-1}, 2^{-i}\right], \quad d_{i} \text { is a constant with }\left|d_{i}\right| \leq C \epsilon
$$

and similarly on $\left[1-2^{-i}, 1-2^{-i-1}\right]$. In other words, $\phi$ is constructed so that $\phi$ is continuous, $\nabla \phi=\nabla \tilde{\phi}$ on $J_{i}$ and

$$
1-C \epsilon \leq \frac{\int_{\Omega}|\phi|^{2}}{\int_{\Omega}|\tilde{\phi}|^{2}} \leq 1+C \epsilon .
$$

Now we will obtain an estimate on the first eigenvalue $\lambda$ ((b) of Theorem 1.1) using a function perturbed from $\phi$. On $J_{i}$,

$$
\begin{aligned}
|\nabla \phi|=|\nabla \tilde{\phi}| & =|\partial \tilde{\phi} / \partial x| \\
& \leq\left(1+\left|\partial_{s} y_{j}\right|\right)\left|\nabla u\left(s, f\left(s, \tilde{t_{1}}, \ldots, \tilde{t}_{n-1}\right)\right)\right| \\
& \leq\left(1+C 2^{i} \epsilon\right) v(s),
\end{aligned}
$$

where $v(s)$ is a one-dimensional function in $\Omega$ such that

$$
v(s)=\left|\nabla u\left(s, f\left(s, \tilde{t_{1}}, \ldots, \tilde{t}_{n-1}\right)\right)\right| .
$$

Hence

$$
\begin{aligned}
\int_{\Omega}|\nabla \phi|^{2} & =\int_{\Omega\left(\cup_{1 \leq i<k_{0}} J_{i}\right)}|\nabla \phi|^{2} \\
& \leq \int_{\Omega} v^{2}+\sum_{i=1}^{k_{0}-1} C \epsilon 2^{i} \int_{\Omega\left(J_{i}\right)} v^{2} \\
& \leq \int_{\Omega} v^{2}+C \sum_{k=1}^{k_{0}-1} \epsilon 2^{-i} \int_{\Omega\left(J_{i}\right)} 1 \\
& \leq \int_{\Omega}|\nabla u|^{2}+C \epsilon|\Omega| \\
& \leq(1+C \epsilon) \int_{\Omega}|\nabla u|^{2},
\end{aligned}
$$

where $\Omega\left(J_{i}\right):=\Omega \cap\left\{x \in J_{i}\right\}$, the second inequality follows from Corollary 2.4, the third inequality follows from (5.1) and the last inequality follows from the Remark 
and the normalization $\max u=1$. On the other hand, by (5.2), Corollary 2.5] and (5.3)

$$
1-C \epsilon \leq \frac{\int_{\Omega} \phi^{2}}{\int_{\Omega} u^{2}} \leq 1+C \epsilon .
$$

Therefore (5.4) and (5.5) imply

$$
\frac{\int_{\Omega}|\nabla \phi|^{2}}{\int_{\Omega} \phi^{2}} \leq(1+C \epsilon) \frac{\int_{\Omega}|\nabla u|^{2}}{\int_{\Omega} u^{2}} .
$$

Since $\phi$ does not satisfy the constraint $\int_{\Omega} \phi=0$, we construct $\psi$ by perturbing $\phi$, which satisfies $\int_{\Omega} \psi=0$ as well as (5.6). From (5.2) and $\int_{\Omega} u=0$,

$$
\left|\int_{\Omega} \phi\right| \leq C \epsilon|\Omega|
$$

Let $z=\left(z_{1}, \ldots, z_{n}\right)$ be a point on

$$
\left\{\left(s, f\left(s, f\left(s, \tilde{t_{1}}, \ldots, \tilde{t}_{n-1}\right)\right)\right): 0 \leq s \leq 1\right\}
$$

such that $u(z)=0$. (Note that $z=\left(z_{1}, \ldots, z_{n}\right) \in \Lambda$.) Then by Corollary 2.5, $c_{1} \leq z_{1} \leq 1-c_{1}$ for a dimensional constant $c_{1}>0$. This bound on $z_{1}$ and (5.7) imply that there exists $c_{0}$ such that $\psi(x):=\phi(x)+c_{0} \epsilon\left(x-z_{1}\right)^{+}$satisfies $\int_{\Omega} \psi=0$ with $\left|c_{0}\right|<C$ for a dimensional constant $C$. Observe that (5.6) also holds for $\psi$, and thus

$$
\mu_{1} \leq \frac{\int_{\Omega}|\nabla \psi|^{2}}{\int_{\Omega} \psi^{2}} \leq(1+C \epsilon) \frac{\int_{\Omega}|\nabla u|^{2}}{\int_{\Omega} u^{2}}=(1+C \epsilon) \lambda,
$$

which proves part (b) of Theorem 1.1.

For the proof of part (a), we first show that the projection of the nodal set $\Lambda$ of $u$ onto the $x$-axis is contained in an interval of length $C \epsilon$ and then we locate $\Lambda$ near the zero of $\phi_{1}$. Let $p$ be a projection on the $x$-axis. Then $p\left(\bar{\Omega}_{+}\right)$and $p\left(\bar{\Omega}_{-}\right)$ are intervals because the Courant nodal domain theorem [CH, p. 452] implies that $\Omega_{+}$and $\Omega_{-}$are connected. Hence $p(\Lambda)=[a, b]$ for some $a \leq b$. By (5.2),

$$
\max _{[a, b]}|u|<C \epsilon,
$$

and since $\max |\psi-u| \leq C \epsilon$,

$$
\sup _{[a, b]}|\psi|<C \epsilon .
$$


By Corollary 2.5, $\left|\int_{a}^{1} \phi w d s\right|>C \int_{0}^{1} w d s$ for a dimensional constant $C>0$. Hence Lemma 3.6 with (5.8) and (5.9) implies that $b-a<C \epsilon$ for a dimensional constant $C$. In other words, the nodal set $\Lambda$ is contained in an $x$-interval of length $C \epsilon$.

Now, observe

$$
\begin{aligned}
\frac{\int_{z_{1}}^{1} \psi^{\prime 2} w d s}{\int_{z_{1}}^{1} \psi^{2} w d s} \leq(1+C \epsilon) \frac{\int_{z_{1}}^{1} \phi^{\prime 2} w d s}{\int_{z_{1}}^{1} \phi^{2} w d s} \leq(1+C \epsilon) \frac{\int_{\Omega^{\prime}}|\nabla u|^{2}}{\int_{\Omega^{\prime}} u^{2}} \\
\leq(1+C \epsilon) \frac{\int_{\Omega_{+}}|\nabla u|^{2}}{\int_{\Omega_{+}} u^{2}}=(1+C \epsilon) \lambda \leq(1+C \epsilon) \mu_{1},
\end{aligned}
$$

where $\Omega^{\prime}=\Omega \cap\left\{z_{1} \leq x \leq 1\right\}, \psi\left(z_{1}\right)=\phi\left(z_{1}\right)=0$, the second inequality follows from a similar argument as in (5.6) and the third inequality follows from $b-a<C \epsilon$, $|u| \leq 1$ and $|\nabla u| \leq C$. By Lemma 3.5, $z_{1} \leq s_{1}+C \epsilon$. By a similar argument on the interval $\left[0, z_{1}\right]$, we obtain $s_{1}-C \epsilon \leq z_{1}$. Since the length of the projection of the nodal set on the $x$-axis is less than $C \epsilon$, part (a) is proved.

Lastly we discuss the general case. For a general domain $\Omega$, let $\left\{\Omega_{k}\right\}_{k}$ be an increasing sequence of smooth domains which converges to $\Omega$ uniformly on each cross section $\Omega_{k}\left(x_{1}\right)$. Let $u_{k}$ be the corresponding first nonzero eigenfunction of $\Omega_{k}$ with $\sup \left|u_{k}\right|=1$. Then by Kröger's theorem (Theorem 2.1), $\sup _{\Omega_{k}}\left|\nabla u_{k}\right|$ is uniformly bounded. Hence there exists a subsequence $\left\{u_{k_{j}}\right\}_{j}$ which converges uniformly to $u$ and $\left\{\lambda_{k_{j}}\right\}_{j}$ converges to $\lambda$ as $j \rightarrow \infty$. On the other hand, since the volume $w_{k}(x)$ of $\Omega_{k}(x)$ uniformly converges to $w(x)$, we may assume that $\left\{\phi_{k_{j}}\right\}_{j}$ converges uniformly to $\phi_{1}$ and $\left\{\mu_{k_{j}}\right\}_{j}$ converges to $\mu$ as $j \rightarrow \infty$. Now by the nondegeneracy of $\phi_{k_{j}}$ (Lemma 3.7) and the nondegeneracy of $u_{k_{j}}$ in the scale $\epsilon$ (Lemma 3.6), we obtain Theorem 1.1] for $u$ and $\lambda$.

\section{REFERENCES}

[BQ] D. Bakry, Z. Qian, Some new results on eigenvectors via dimension, diameter, and Ricci curvature, Adv. Math., 155(2000), 98-153. MR.1789850 (2002g:58048)

[CH] R. Courant, D. Hilbert, Methods of Mathematical Physics. Vol. I, Interscience Publishers, New York (1953). MR0065391 (16:426a)

[GJ1] D. Grieser, D. Jerison, Asymptotics of the first nodal line of a convex domain, Inventiones Math., 125(1996), 197-219. MR.1395718(97d:35033)

[GJ2] D. Grieser, D. Jerison, The size of the first eigenfunction of a convex planar domain, $J$. Amer. Math., 11(1998), 41-72. MR1470858 (2000f:35034)

[K1] P. Kröger, On the spectral gap for compact manifolds, J. Differential Geom., 36(1992), 315-330. MR.1180385 (94g:58236)

[K2] P. Kröger, On the ranges of eigenfunctions on compact manifolds, Bull. London Math. Soc., 30(1998), no.6, 651-655. MR1642763 (99j:58191)

[J1] D. Jerison, Locating the first nodal line in the Neumann problem, Trans. Amer. Math. Soc., 352(2000), 2301-2317. MR 1694293(2001b:35234)

[J2] D. Jerison, The diameter of the first nodal line of a convex domain, Annals of Math., 141(1995), 1-33. MR.1314030 (95k:35148) 
[L] P. Li, A lower bound for the first eigenvalue of the Laplacian on a compact manifold, Indiana U. Math. J., 28(1979), 1013-1019. MR551166 (81j:58084)

[ZY] J. Q. Zhong, H. C. Yang, On the estimate of the first eigenvalue of a compact Riemannian manifold, Sci. Sinica Ser. A, 27(12)(1984), 1265-1273. MR.794292(87a:58162)

Department of Mathematics, University of Arizona, Tucson, Arizona 85721

E-mail address: schoi@math.arizona.edu

Department of Mathematics, Massachusetts institute of Technology, Cambridge, Massachusetts 02139

E-mail address: jerison@math.mit.edu

Department of Mathematics, University of California, los Angeles, Los Angeles, CALIFORNIA 90095

E-mail address: ikim@math.ucla.edu 